\title{
¿IDENTIDAD CRISTIANA ÉTNICA? EL CASO DE DIOGN. 1.1
}

Fecha de recepción: 16 de enero de 2019

Fecha de aceptación y versión final: 25 de febrero de 2019

RESUMEN: El carácter étnico del cristianismo primitivo es un tema debatido en la

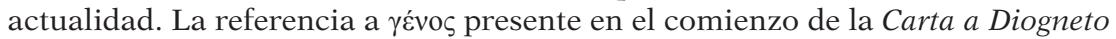
es aducida con frecuencia para defender una comprensión racial/étnica de la identidad cristiana. Para algunos autores esta interpretación se apoya en las referencias a la expresión «tercer $\gamma \varepsilon ́ v o \varsigma / g e n u s »$ que se encuentra en otros escritores cristianos anteriores o contemporáneos a Diogn. El presente trabajo aboga

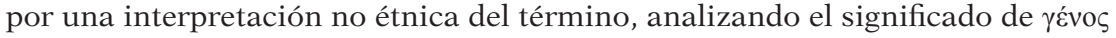

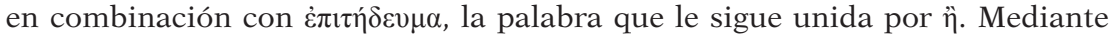
ejemplos tomados de la literatura griega clásica y cristiana, se sostiene que con

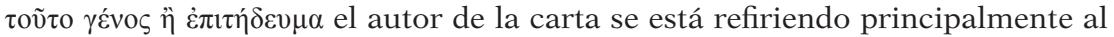
modo de vida de los cristianos en la sociedad en que habitan sin connotaciones étnicas.

PALABRAS CLAVE: etnicidad; identidad; Carta a Diogneto; tercera raza; literatura cristiana primitiva; modo de vida cristiano.

* Facultad de Teología. Universidad de Navarra: jchapa@unav.es; ORCID: https://orcid.org/0000-0001-7345-0625 


\section{Ethnic Christian Identity? The Case of Diogn. 1.1}

ABSTRACT: The ethnic character of early Christianity is a disputed subject. The reference to $\gamma \varepsilon \dot{v} v \varsigma$ at the beginning of the Letter to Diognetus is often mentioned in the debate to support a racial/ethnic understanding of Christian identity. For some scholars this interpretation would seem to be supported by the expression "third $\gamma \varepsilon$ vo /genus», which is found in Christian writers prior or contemporary to Diogn. The present paper advocates a non-ethnic interpretation of the term, by analysing the meaning of $\gamma \varepsilon \dot{v}$ o

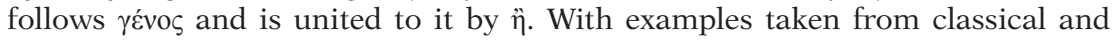

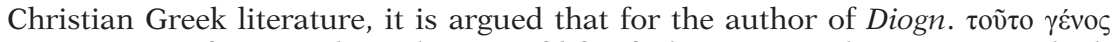

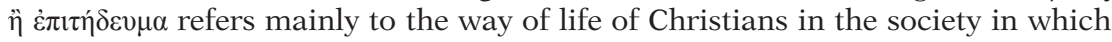
they live, with no ethnic connotations.

KEY WORDS: ethnicity; identity; Letter to Diognetus; third race; early Christian literature; Christian way of life.

$$
* * *
$$

El tema de la multiplicidad étnica y cultural, tan en boga hoy, ha llegado también con fuerza a los estudios sobre los orígenes cristianos. No faltan los trabajos en los que se considera y discute hasta qué punto la etnicidad es un elemento fundamental para expresar la identidad de los primeros seguidores de Jesús. Siguiendo la estela de autores que estudiaron la identidad étnica en el mundo clásico ${ }^{1}$, en las últimas décadas se ha escrito abundantemente sobre esta cuestión en relación con el cristianismo inicial. Entre la amplia producción destacan, por citar solo algunas, las obras de Lieu, Buell o Horrell ${ }^{2}$. Las conclusiones a las

1 Edith Hall, Inventing the Barbarian: Greek Self-Definition through Tragedy (Oxford: Oxford University Press, 1991); Siân Jones, The Archeology of Ethnicity: Constructing Identities in the Past and the Present (New York: Routledge, 1997); Jonathan M. Hall, Ethnic Identity in Greek Antiquity (Cambridge: Cambridge University Press, 1997); Benjamin Isaac, The Invention of Racism in Classical Antiquity (Princeton: Princeton University Press, 2004).

2 Mark G. Brett, ed., Ethnicity and the Bible (Boston: Brill, 1996); Gay L. Byron, Symbolic Blackness and Ethnic Difference in Early Christian Literature (NewYork: Routledge, 2002); Judith M. Lieu, Neither Jew nor Greek? Constructing Early Chris-

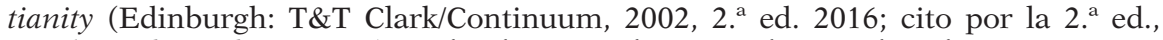
London: Bloomsbury, 2016); Ead., Christian Identity in the Jewish and Graeco-Roman World (New York: Oxford University Press, 2004); Denise K. Buell, Why This New Race: Ethnic Reasoning in Early Christianity (New York: Columbia University Press, 2005); Shaye J. D. Cohen, Why Aren't Jewish Women Circumcised? Gender and Covenant in Judaism (Berkeley: University of California Press, 2005); David G. Horrell, 
que llegan estos autores no son homogéneas, pues los propios contextos sociopolíticos y religiosos pesan mucho a la hora de realizar un análisis de este tipo ${ }^{3}$. Y no faltan tampoco voces críticas que consideran que el concepto de identidad étnica, acuñado en mitad del siglo $\mathrm{XX}^{4}$, no es más que un discurso social que poco tiene que ver con la realidad histórica de hace dos mil años y que se aplica a esa realidad de forma anacrónica ${ }^{5}$.

Sea como fuere, se observa el deseo de considerar a los cristianos como un grupo cuya identidad se expresaba en términos de etnia y raza, conforme a un carácter inclusivo que no era tan diferente de la comprensión que tenían los judíos de su propia identidad. La provocadora obra de Denise Buell, Why This New Race: Ethnic Reasoning in Early Christianity (2005), ha sido pionera en este campo. En ella, la autora analiza los modos en que los escritores cristianos de los tres primeros siglos se situaban dentro del terreno étnico, racial y religioso de los discursos de identidad del mundo grecorromano, e intenta mostrar que esas comprensiones están en contra de las concepciones habituales sobre «raza» y «religión» que aparecen en el ámbito académico actual aplicadas a los

Becoming a Christian. Essays in 1 Peter and the Making of Christian Identity (London: Bloomsbury T\&T Clark, 2013). Y más recientemente: David G. Horrell \& Katharine M. Hockey, eds., Ethnicity, Race, Religion. Identities and Ideologies in Early Jewish and Christian Texts, and in Modern Biblical Interpretation (London: T\&T Clark, 2018).

3 Ciertamente, es también inevitable que ocurra en quien escribe estas páginas, pues todos estamos condicionados por la propia formación cultural.

4 Ver John Hutchinson \& Anthony D. Smith, eds., Ethnicity (Oxford: Oxford University Press, 1996), 4-7. Para una presentación sintética, ver también Dennis C. Duling, "2 Corinthians 11:22: Historical Context, Rhetoric, and Ethnic Identity", en The New Testament and Early Christian Literature in Greco-Roman Context: Studies in Honor of David E. Aune, ed. John Fotopoulos, Novum Testamentum, Supplements 122 (Leiden: Brill, 2006), 85, quien resume lo que podrían ser los rasgos fundamentales de etnicidad sintetizando la aportación de otros autores: 1 . Nombre propio que identifica y expresa la "esencia» de la comunidad. 2. Mitos de un antepasado común relacionados con un tiempo y espacio distantes que dan un sentido de parentesco ficticio. 3. Recuerdos compartidos del "pasado", incluyendo héroes y sucesos, y sus conmemoraciones. 4. Rasgos fenotípicos (genéticos). 5. Alguna conexión con una patria, sea física o simbólica. 6. Cultura común, incluyendo lenguaje y religión. 7. Sentido de solidaridad con una población étnica.

5 Ver Erich S. Gruen, "Christians as a 'Third Race'”, en Christianity in the Second Century. Themes and Developments, ed. James Carleton Paget y Judith Lieu (Cambridge: Cambridge University Press, 2017), 235-249 y su recensión al libro de Buell, "Why This New Race”, Catholic Biblical Quaterly 72 (2010): 365-367. 
primeros siglos de cristianismo ${ }^{6}$. El punto de partida desde el que argumenta Buell es la Carta a Diogneto. La autora señala que la pregunta que, según el autor de la carta, se hace Diogneto: «¿Por qué esta nueva raza?» se ha contestado tradicionalmente con una respuesta, a su juicio, simplista: el cristianismo no es una raza sino una religión universal (conforme a la comprensión paulina, según la cual en la fe en Cristo ya no hay distinción de razas ni de pueblos, ni de judíos ni de griegos) ${ }^{7}$. Buell, en cambio, cuestiona esa respuesta y trata de mostrar que la identidad del cristianismo como una «no-raza» es algo que responde a sentimientos modernos y no a la realidad histórica, y sostiene que los cristianos argumentaban precisamente en términos de etnicidad. Para demostrarlo, se propone descubrir qué dicen los primeros textos cristianos si se analizan desde este punto de vista. En concreto, en relación al texto de Diogn. el presupuesto del que parte Buell es el siguiente:

"Before addressing the specifics of the Christians in question, the narrator first offers a criticism of "Greeks" and "Jews", focusing specifically on their respective practices. Thus, the structure of the narrative makes Christians comparable with and parallel to Greeks and Jews, and then portrays Greekness and Jewishness especially via "religious" ideas and actions. Rhetorically, this positions Christians as comprising a genos, like Greeks and Jews; moreover, it implies that the salient content of a genos for this text is "religion", depicted in terms of both appropriate practices and the appropriate understanding of

6 Parte de los presupuestos de Buell se pueden encontrar en estas afirmaciones: «[M]y methodological and sociopolitical commitments [...] condition the possibilities for and importance of interpreting some forms of early Christian imagination and practice as "ethnic reasoning". I argue that by strategically using the modern categories of race and ethnicity to speak about early Christian self-definition, we will be better able to resolve a problematic paradox in the way these concepts have informed historical reconstructions of early Christians. Specifically, I challenge the view that ethnicity and race were irrelevant to early Christians -an argument that has been used to accomplish important modern antiracist work yet relies on and perpetuates anti-Judaism in the process. To support an interpretation of Christianity that can help end both racism and anti-Judaism, I revisit scholarship and early Christian texts that destabilize the prevailing view that Christian universalism can be understood as mutually exclusive with "particularity" -a split that is often correlated with the nonethnic/ ethnic binary» (p. x) (el subrayado es mío).

7 Cf. Col 3,11: «para quien no hay griego o judío, circuncisión o no circuncisión, bárbaro o escita, siervo o libre, sino que Cristo es todo en todos»; Gal 3,28: «Ya no hay diferencia entre judío y griego, ni entre esclavo y libre, ni entre varón y mujer, porque todos vosotros sois uno solo en Cristo Jesús». 
the object of worship. This structure raises the possibility that this text is defining "race" in quite a different way than is familiar to most modern readers ${ }^{8}$.

Para Buell, si la cuestión que trata la carta se sintetiza en: ¿qué hace distinto al cristiano?, el narrador contesta que lo que le distingue es el lenguaje, las costumbres y el lugar de residencia (no el origen), es decir, elementos constitutivos del lenguaje étnico. La autora entiende así que para Diogn. el cristianismo es una raza (tal como ya en su opinión lo habían afirmado Arístides y Atenágoras) concordando de este modo con la tradición judía, en la cual se utiliza la noción de raza para indicar un pueblo que abraza a todos y que se articula con la visión de una ciudadanía universal en cuanto pueblo ${ }^{9}$. En contra de la extendida comprensión contemporánea de que el cristianismo constituye una novedad y que se debe clasificar como «religión», rompiendo la conexión con rasgos étnicos y raciales -y en contra de la idea de que los cristianos de hoy están reinventando su pasado para salir al paso de las necesidades del presente $-^{10}$, Buell considera que los cristianos acudieron habitualmente a la terminología de "raza» para caracterizar su propia identidad ${ }^{11}$. Ahora bien, ¿se debe entender yévos como lo hace esta autora? Conviene estudiarlo, aunque la obra de Buell ha sido alabada por su agudeza y novedad, también se ha dicho de ella que sus argumentos deben todavía encontrar más apoyo en análisis filológicos ${ }^{12}$.

8 Buell, Why This New Race, 30.

9 Ibid., 33.

10 Ibid., 67.

11 En la cuestión del $\gamma \varepsilon ́ v o \varsigma$, ver Denise K. Buell, "Rethinking the Relevance of Race for Early Christian Self-Definition", Harvard Theological Review 94 (2001): 461462; Ead., "Race and Universalism in Early Christianity", Journal of Early Cristian Studies 10 (2002): 429-468. La autora insiste en estos trabajos en que etnicidad y raza han sido de hecho fundamentales en las diversas fórmulas de autodefinición de los primeros cristianos. Ver también Judith M. Lieu, "Race of the God-Fearers", The Journal of Theological Studies New Series 46 (1995): 483-501 = Neither Jew nor Greek?, 66-85; Ead., Christian Identity, 239-268.

12 Ver por ej. la recensión de Margaret M. Mitchell, en History of Religions 48 (2008): 175, y Aaron P. Johnson en Bryn Mawr Classical Review 2006.02.31 http://bmcr. brynmawr.edu/2006/2006-02-31.html, consultado el 27.9.2018. También se le ha criticado la poca claridad de lenguaje. Así Erich Gruer en su recensión (Catholic Biblical Quaterly 72 [2010]: 367) recoge como ejemplo de esa oscuridad la siguiente frase: «The conceptualization that I have adopted in this book of race and ethnicity as being characterized by both fixity and fluidity suits the prismatic approach because it 
Buell no es la única que se ha fijado en el texto de Diogn. desde esta perspectiva. Judith Lieu, quien en diversos trabajos ha estudiado el tema de la identidad de los cristianos en el siglo II, recientemente ha vuelto sobre este texto ${ }^{13}$. Sin embargo, lo hace de una manera mucho más matizada que Buell ${ }^{14}$. Coincide con ella en que el punto de partida es la supuesta pregunta de Diogneto sobre qué tipo de $\gamma \varepsilon ́ v o \varsigma$ es esta nueva religión y, al igual que Buell, lo vincula a Diogn. 5.1.5 donde se dice que los cristianos no se distinguen de los otros pueblos por su país, lengua o vestido (o costumbre), porque su patria es un lugar extranjero y toda patria es extranjera. Ciertamente, admite que el empleo de la palabra yévos da pie a una intepretación étnica, pero señala que este uso es a la vez y paradójicamente trascendente ${ }^{15}$. Lieu argumenta que la $\theta \varepsilon 0 \sigma \varepsilon ́ \beta \varepsilon ı \alpha$ es el punto central en la carta y que se caracteriza por tres elementos: 1) la identidad de Dios al que los cristianos dan culto; 2) un tipo de amor que

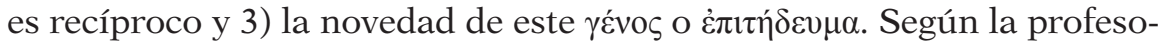
ra británica, para alguien que no era cristiano, los dos primeros puntos asemejarían la religión cristiana a otra más. Sin embargo, el tercer elemento sirve para sintetizar los otros dos y distinguir al cristianismo ${ }^{16}$. Así pues, tras analizar los dos primeros, al estudiar el tercero recuerda la doble interpretación que se ha dado a $\gamma \varepsilon ́ v o \varsigma$ en este pasaje: como tipo/

highlights the shapeshifting instantiations of these complexly interrelated concepts without insisting that they have any intrinsic essence» (Buell, Why This New Race, 168).

13 Judith M. Lieu, "Identity Games in Early Christian Texts: The Letter to Diognetus", en Ethnicity, Race, Religion, ed. Horrell \& Hockey, 59-72. Anteriormente había estudiado la cuestión en Judith M. Lieu, "The Forging of Christian Identity", Mediterranean Archaeology 11 (1998): 71-82 (= "The Forging of Christian Identity and the Letter to Diognetus", en Neither Jew nor Greek?, 185-203). Aquí trata de la identidad cristiana en Diogn.

14 De hecho, a la pregunta que plantea el autor de la carta sobre la novedad del cristianismo que da pie a la argumentación de Buell, vista más arriba, Lieu, Christian Identity, 85-86, afirma que el autor de la carta no da respuesta a esa pregunta, sino responde solo «by demonstrating the vacuity of the pagan and Jewish alternatives, and by marvelling at God's patience until the present».

15 Lieu, "Identity Games", 60.

16 Ibid., 62. En esta línea, anteriormente, Lieu, "The Race of the God Fearers", 488 (= Neither Jew nor Greek?, 71), había argumentado que el tema de la carta se centra en cómo, debido al rechazo de los que son considerados dioses por los griegos y de la superstición $-\delta \varepsilon \imath \_$$\delta \alpha \mu o v i ́ \alpha-$ de los judíos, los cristianos son constituidos en una nueva raza ( $\gamma \varepsilon \dot{v} \vee \varsigma)$ o práctica $\left(\dot{\varepsilon} \pi \iota \eta^{\delta} \delta \varepsilon \mu \mu \alpha\right)$. 


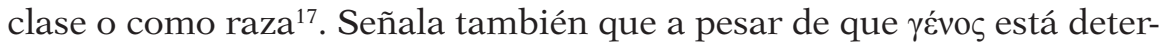

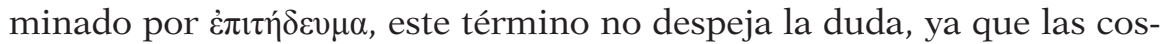
tumbres, el lenguaje y modo de vida permitían a un bárbaro ser griego, o a un griego ser judío. Esta versatilidad se muestra, por ejemplo, en un texto de Luciano (Scyth. 1-3), según el cual queda claro que las costumbres tienen que ver con la raza, y otro de Galeno (De sanitate 6,51), donde menciona que entre su público se incluyen «los que la naturaleza hizo

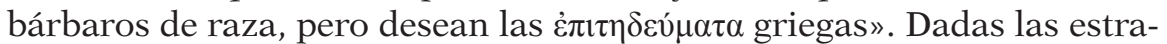
tegias retóricas en juego, afirma Lieu, Diogn. no deja claro hasta qué punto las prácticas sociales no entran también en un modo de razonar étnico, e indica que los precedentes de Arístides y de la Predicación de Pedro que habitualmente se aducen para defender una intepretación ét-

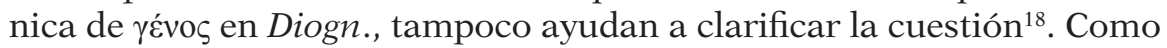
consecuencia, considera que el autor de Diogn. ni afirma ni niega las categorías de "raza y modo de vida» ni las ha reinterpretado conforme a unas categorías de ciudadanía transfigurada, sino que el tema clave de la carta es el de la novedad del cristianismo, que no se expresa en términos de profecía o plan salvífico, sino en relación a la persona que debe renovarse. El fundamento de todo ello se encuentra en la realidad de que la

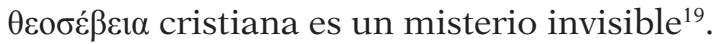

Por otra parte, Samuel Vollenweider se ha fijado también en la Carta a Diogneto como clave para entender aspectos étnicos presentes en las cartas paulinas ${ }^{20}$. Admitiendo que en Diogn. se puede dar la impresión de que se comprende el cristianismo como «tercera raza», sin embargo, afirma que en este escrito hay una tendencia a ir más allá de una definición étnica de cristianismo. Vollenweider piensa que la paradoja que presenta la carta sobre la no diferenciación entre los cristianos y los demás y al mismo tiempo caracterizar a los seguidores de Cristo por tener una «etnicidad» propia es resuelta por la retórica,

17 Lieu, "Identity Games", 69. En Lieu, Neither Jew nor Greek?, 197, se había referido a $\gamma \varepsilon ́$ vo como un término ambiguo.

18 Lieu, "Identity Games", 70. Un ejemplo claro de las dificultades que acarrea

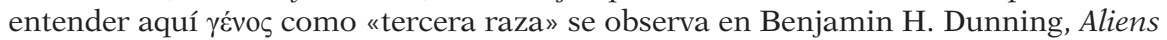
and Sojourners: Self as Other in Early Christianity, Divinations: Rereading Late Ancient Religion (Philadelphia: University of Pennsylvania Press, 2009), 67-68.

19 Lieu, "Identity Games", 71.

20 Samuel Vollenweider, “Are Christians a New 'People'?: Detecting Ethnicity and Cultural Friction in Paul's Letters and Early Christianity", Early Christianity, 8 (2017): 293-308. 
que reduce la fuerza de las categorías étnicas: «La etnicidad no es sino un modelo formativo entre otros en la construcción de la identidad cristiana, complementada por otros grupos metafóricos que parecen ser más centrales a la autodefinición cristiana» ${ }^{21}$. Como se ve, el tema de la etnicidad está más matizado, aunque se da por supuesto que se emplea el lenguaje étnico.

A la luz de lo expuesto, deseo en estas páginas fijarme en la pregunta inicial de la Carta a Diogneto y tratar de ver hasta qué punto la expresión

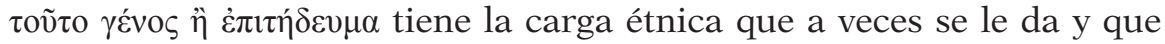
a menudo condiciona el modo de entender el tipo de cristianismo que trasluce este escrito.

\section{TIPO Y ESTILO DE VIDA}

Las traducciones de los términos en las diversas ediciones de Diogn. apuntan mayoritariamente a la concepción de $\gamma \varepsilon \dot{\varepsilon} v \varsigma \varsigma$ como raza y de $\dot{\pi} \pi \imath \eta^{\prime} \delta \varepsilon v \mu \alpha$ como práctica, modo de vida o costumbres. Así, en al-

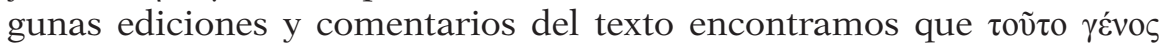

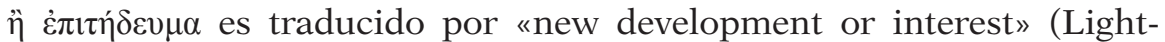
food-Harmer, 1907), «this new race of men or mode of living» (Meecham, 1949), "ce peuple nouveau -ce nouveau mode de vie» (Marrou, 1951), «raza, o nuevo género de vida» (Ruiz Bueno, 1965), «stirpe o pratica di vita» (Norelli, 1991), «nueva raza o manera de vivir» (Ayán, 2000), «neue Geschlecht oder diese neue Lebensweise» (Lona, 2001), «this new race or way of life» (Ehrman, 2003; Jefford, 2013). Como se observa, los términos oscilan entre una concepción abstracta de $\gamma \varepsilon ́ v o \varsigma$ desligada de connotaciones raciales (solo representada por Lightfood-Harmer) a un predominio generalizado de una interpretación con connotaciones étnicas. ¿Resulta, sin embargo, tan claro que este término tenga el sentido que mayoritariamente se le da? Es posible, pero, al menos, $\gamma \varepsilon ́ v o \varsigma$ debe ser contrastado con $\dot{\varepsilon} \pi \tau \eta ́ \delta \varepsilon v \mu \alpha$, que va unido a este por una conjunción disyuntiva y que hace que los dos vocablos no se presenten como alternativa, sino que el segundo aparezca más bien como explicativo del primero, al modo de una glosa o un suplemento apropiado ${ }^{22}$.

\footnotetext{
$21 \quad$ Ibid., 297.

22 Gruen, 239-240. El autor remite a Horacio E. Lona, An Diognet. Übersetzt und erklärt (Freiburg: Herder, 2001), 78-79 y añade: «Alternatively, one might wish to see 


\section{1. ¿LA RAZA DE LOS CRISTIANOS?}

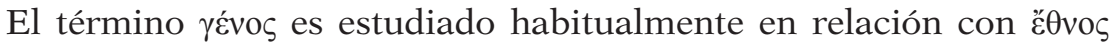
y $\lambda \alpha o^{23}{ }^{23}$. Como es bien sabido, en griego clásico हैं $\theta$ vo normalmente se empleaba para designar un grupo de gente, pero también podía indicar un tipo de seres que participan de una identidad común, humana o ani$\mathrm{mal}^{24}$. Asimismo, se utilizaba para designar a grupos de gente extranjera, a un grupo específico de personas (clase, tribu, casta) o incluso a una provincia $^{25}$. Por su parte, $\gamma \varepsilon ́ v o \varsigma$ es más especializado. Aunque se podía también aplicar a grupos humanos y animales, a tipos y clases de cosas, así como a grupos raciales o étnicos, el término se fija más en la noción de descendencia común (aunque no necesariamente real) ${ }^{26} . \lambda \alpha$ cós, finalmente, parece que siempre se refiere a grupos de gente, a veces en cuanto distintos de sus dirigentes ${ }^{27}$.

the statement as reflecting uncertainty as to just how Christianity should be defined. But that does not suit the context or the thrust of the passage». Por su parte, Clayton N. Jefford, The Epistle to Diognetus (with the Fragment of Quadratus). Introduction, Text and Commentary (Oxford: Oxford University Press, 2013), 201, comenta que el

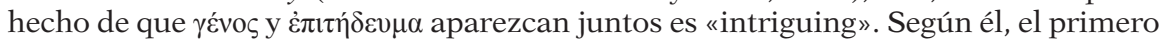
refleja el surgir en el siglo II de opiniones sobre el cristianismo como un nuevo orden de una realidad existencial.

23 Para un resumen de los significados David G. Horrell, “'Race', 'Nation', 'People': Ethnic Identity-Construction in 1 Peter 2:9”, New Testament Studies 58 (2011): 123-143, esp. 125-130, reproducido y ampliado en Becoming a Christian, 133-163, esp. 133-144. Ver también Aaron P. Johnson, Ethnicity and Argument in Eusebius' Praeparatio Evangelica, Oxford Early Christian Studies (Oxford: Oxford University Press, 2006), 25-54.

24 Hall, Ethnic Identity in Greek Antiquity, 34-35. Este grupo puede estar formado por personas o animales que viven o están en compañía de otros (incluido una bandada de pájaros o un grupo de gusanos). Ver LSJ, s.v. č $\theta v o s, 1$.

25 H. G. Liddell, Robert Scott \& H. Stuart Jones, Greek-English Lexicon, $9^{\text {th }}$ ed. with revised supplement (Oxford: Clarendon Press, 1996), s.v. है $\theta$ vos 2 y 3; Walter Bauer, William F. Arndt \& F. William Gingrich, A Greek-English Lexicon of the New Testament and Other Early Christian Literature, $3^{\text {rd }}$ ed. (Chicago: University of Chicago Press, 2000), s.v. है $\theta$ vo $276 \S 2$.

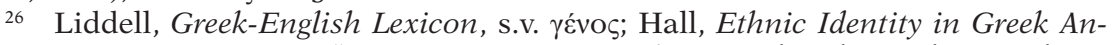

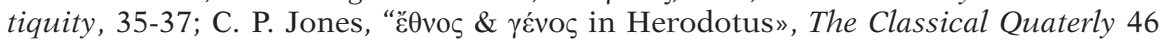
(1996): 317.

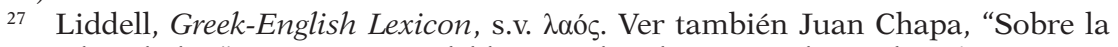
relación laos-laikos", en La misión del laico en la Iglesia y en el mundo, ed. José Luis Illanes et al. (Pamplona: Eunsa, 1987), 197-212. 


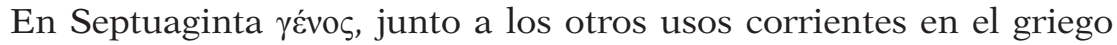
clásico, se utiliza sobre todo para designar al pueblo de Israel, en sentido parecido al de $\lambda \alpha o ́ s$, y es el más corriente desde época macabea ${ }^{28}$. En cambio, $\check{\varepsilon} \theta v \eta$ se emplea sobre todo para designar a las «naciones», es decir, a los que no pertenecen al pueblo (aunque en ocasiones los tres términos son sinónimos y $\check{\varepsilon} \theta v \eta$ se utiliza también para designar al pueblo de Israel ${ }^{29}$. La idea de una descendencia común es especialmente importante en $\gamma \varepsilon ́ v o s$, en cuanto que subraya lo que más adelante podrá ser entendido como una designación étnica o racial. Por eso, Horrell señala que la presencia de los tres términos en 1Pe 2,9 es significativa ya que no aparecen juntos en ningún otro lugar del N. T. (salvo quizá en Mt 21,43) y el texto a primera vista parece subrayar el carácter étnico o étnico racial de la identidad cristiana. En todo caso, es el único lugar neotestamentario en el que se designa a los miembros de la Iglesia है $\theta v o \varsigma$, y a la

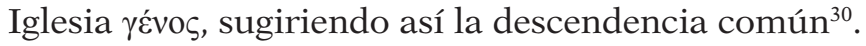

En la primitiva literatura cristiana la primera mención clara de $\gamma \varepsilon \dot{v}$ o referida a los cristianos se encuentra en el Martirio de Policarpo 3.2. Se trata del pasaje en que se narra cómo la gente que esperaba en el circo a que llegara el obispo se quedó admirada al ver «el coraje de la santa

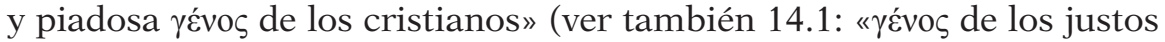

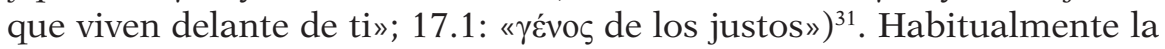

28 Según Johan Lust, Erik Eynikel, y Katrin Hauspie, Greek-English Lexicon of

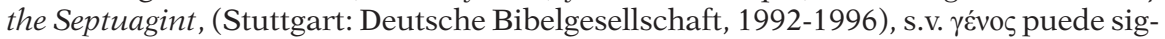
nificar: tipo, clase (Gn 1,11); raza (Lv 21,17); especie (Gn 8,19); nación (Jer 38(31),1);

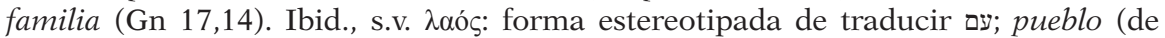
Israel) (en oposición a otras naciones, גוים ž̀ ž $\theta v \eta)$ (Ex 12,33); hombres, pueblo (Gn 14,16); pueblo, ejército (Jos 10,5); pueblo (en oposición a sacerdotes y levitas) 1 Es 5,45; un pueblo (Gn 25,23).

29 Lust, Greek-English Septuagint Lexicon, s.v. हैं vvos: forma estereotipada de traducir ג nación, pueblo (Gn 10,5); no-judios, gentiles (Ps 2,1); la nación judía (designada por los gentiles) (2 Mc 11,25).

30 Horrell, “'Race', 'Nation', 'People”, 129-130; Becoming a Christian, 141-142. Sostiene además que es probable que a partir de este texto se desarrollara el lenguaje de raza aplicado a los cristianos: «Just as 1 Peter represents the first attempt to claim

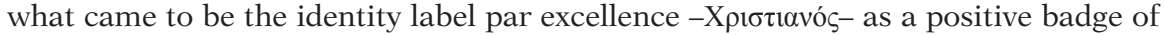
self-identity..., so too it represents the first move to designate Christians explicitly as a $\gamma \varepsilon \dot{v} 0 \varsigma$, a move that was of considerable significance in the evolution of Christian identity discourse» (ibid., 163). Sin embargo, se podría aducir que el hecho de que el texto de 1 Pe sea una cita quita peso a esta comprensión.

31 Candida Moss, "On the Dating of Polycarp: Rethinking the Place of the Martyrdom of Polycarp in the History of Christianity”, Early Christianity 1 (2010): 539-574, 
expresión en 3.2 se traduce por «raza de los cristianos». Pero el término en sí mismo no tiene por qué implicar un sentido étnico. Puede indicar simplemente el carácter de los cristianos, en cuanto un grupo de gente determinado $^{32}$. El siguiente autor que parece utilizar el lenguaje étnico para hablar de la identidad de los cristianos como grupo distinto del de los judíos es Justino ${ }^{33}$. El amplio uso del concepto «pueblo de Dios» para referirse a los cristianos que aparece en el Diálogo con Trifón y el empleo

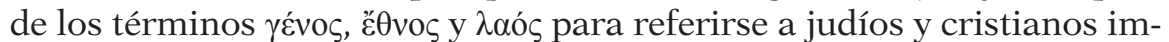
plica que el discurso étnico es importante en su forma de razonar en su confrontación con el pueblo judío ${ }^{34}$. Justino habla de que judíos y cris-

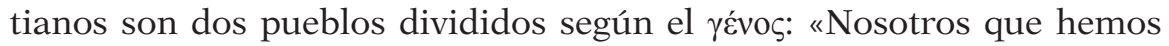
salido, como piedra de una cantera, del vientre de Cristo, somos el ver-

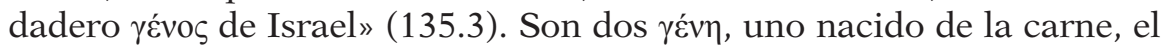
otro de la fe y del espíritu (135.6), siendo Cristo el primogénito y origen

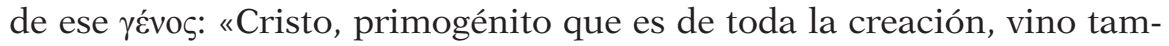
bién a ser principio de un nuevo $\gamma \varepsilon ́ v o \varsigma$, por él regenerado con el agua» (138.2). No obstante, en la Apología, Justino no se refiere a los cristianos

ha defendido que es una falsificación del siglo III. Sin embargo, habitualmente se considera una obra de la segunda mitad o finales del siglo II, aunque eso no significa que haya sufrido un proceso de elaboración posterior. Ver Paul Hartog, Polycarp's Epistle to the Philippians and the Martyrdom of Polycarp (Oxford: Oxford University Press, 2013), 171-186 y su discusión con Moss (pp. 178-186).

32 Así Gruen, 245. Cf. Lieu, "The Race of the God Fearers", 485-490 (=Neither Jew nor Greek?, 68-74); Ead., Image and Reality: The Jews in the World of the Christians in the Second Century (London: T\&T Clark, 1996), 164-177. Sobre la expresion, ver también Hermas, Sim. 9.17 .5 ( litón a Marco Aurelio (Eusebius, Hist. eccl. 4.26.5), donde se combina $\theta \varepsilon o \sigma \varepsilon ́ \beta \varepsilon 1 \alpha$ con

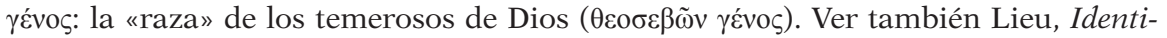
ty Games, 62 y Frederick W. Weidmann, “To Sojourn' or 'To Dwell'?: Scripture and Identity in the Martyrdom of Polycarp", en Reading in Christian Communities: Essays on Interpretation in the Early Church, ed. Charles A. Bobertz \& David Brakke (Notre Dame, IN: University of Notre Dame Press, 2002), 29-40.

33 Ver Buell, Why This New Race, 94-115, para quien, en un largo y cuidado capítulo dedicado a Justino y su Diálogo con Trifón, frente al título de «verdadero Israel» que el filósofo asigna a los cristianos, los judíos encarnan una etnicidad fluida sin límites demasiado fijos en la que los prosélitos se convierten en nativos por la circuncisión. La pretensión cristiana de encarnar de forma auténtica el verdadero pueblo de Dios no solo sirve para excluir otros argumentos de tipo étnico-raciales sino que también utiliza este tipo de retórica para influir en la noción de la salvación individual.

34 Especialmente presentes en Dial. 119.2-4, 120.2, 121.3-5, 123.9, 130.3, 135.3-6. 


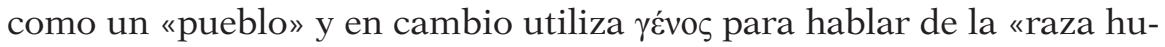
mana». Esta ausencia ha sido explicada como una manera de evitar los riesgos que entrañaba para el autor presentar a los cristianos ante los tribunales romanos como un grupo bien definido ${ }^{35}$. Es posible que fuera así, pero en cualquier caso Justino habla de «toda clase de hombres» para referirse a la multiplicidad de religiones de las que provienen los cristianos y no se cansa de alabar cómo los cristianos pertenecen a todo

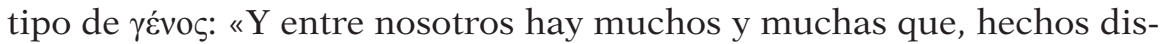
cípulos de Cristo desde niños, permanecen incorruptos hasta los sesenta y setenta años, y yo me glorío de podéroslos mostrar de entre todo

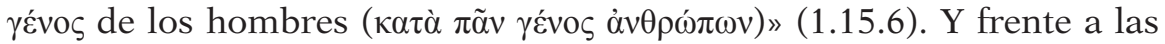
costumbres que los cristianos vivían antes de convertirse dice: «Los que nos odiábamos y matábamos los unos a los otros y no compartíamos el

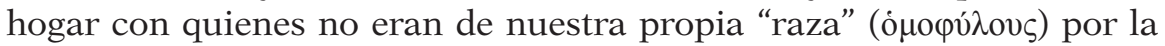
diferencia de costumbres, ahora, después de la aparición de Cristo, vivimos todos juntos» $(1.14 .3)^{36}$. No es fácil precisar el sentido exacto de

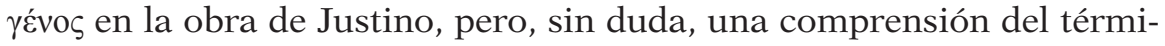
no étnica o racial es cuestionable. Por una parte, porque los cristianos no son del mismo $\gamma \varepsilon \dot{v}$ o rra (1.53.3; cf. 1.25.1; 1.31.7; 1.32.4; 1.39.3; 1.42.4; $1.1 ; 1.40 .7 ; 1.50 .12$; 1.56.1; cf. también Dial. 139.5; 117.5; 121.3) y, por otra, porque son el verdadero $\gamma \varepsilon ́$ vo distinta. El linaje espiritual es real y condición para formar parte de un pueblo, pero es distinto del de la carne. En todo caso, que el cristianismo sea el verdadero Israel no implica que sea otra raza. El lenguaje que emplea Justino puede ser "étnico», pero hace referencia a cuestiones de fe y de observancia de los preceptos de Cristo, no a cuestiones de etnia ${ }^{37}$.

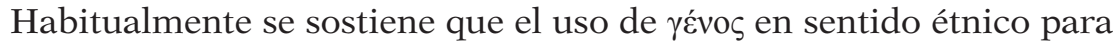
indicar a los cristianos como un grupo distinto de otras razas se da de manera clara en Arístides, Apología 2.3-6. En la versión griega que nos ha llegado se dice: «Porque para nosotros es evidente, joh rey!, que hay

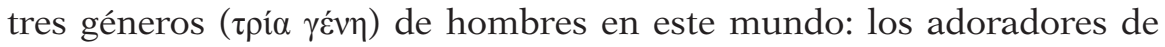
los que entre vosotros llaman dioses, los judíos y los cristianos (oi $\tau \tilde{\omega} v$

35 Oskar Skarsaune, "Ethnic Discouse in Early Christianity", en Christianity in the Second Century, ed. Paget \& Lieu, 259-260.

36 Cf. Peter Lampe, From Paul to Valentinus: Christians at Rome in the First Two Centuries, trad. Michael Steinhauser (Minneapolis: Fortress Press, 2003), 102.

37 Gruen, 245-246. 


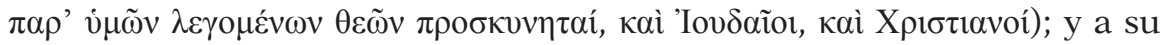
vez, los que veneran a muchos dioses se dividen también en tres géneros

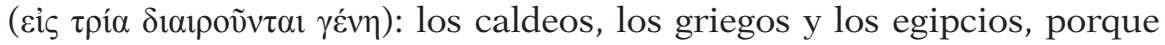
éstos fueron los guías y maestros de las demás naciones en el culto y adoración de los dioses de muchos hombres» ${ }^{38}$. Esta división a veces se aduce como si avalara la comprensión del cristianismo como una «tercera raza» (ver abajo). Independientemente de ello, el problema es que la tradición textual no es uniforme en este pasaje, pues en las versiones siriacas y armenias se dice que son cuatro las clases de hombres (bárbaros, griegos, judíos y cristianos) y no tres como en la tradición griega (seguidores de ídolos -que luego se especifican como caldeos, griegos y egipcios-, judíos y cristianos). Por otra parte, a pesar de las apariencias,

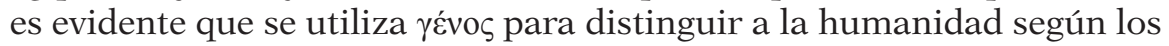
diversos modos de dar culto y no en un sentido étnico-racial ${ }^{39}$.

En esta misma línea se aduce como uso étnico de yévos la expresión

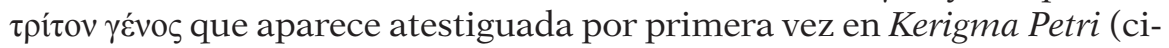
tado por Clemente de Alejandría, Stromata, 6.5.41.6-7), cuando se diferencia a los cristianos de los griegos y judíos: «Pues Dios ha establecido con nosotros una [alianza] nueva [Jer. 31. 31-2]; la [establecida] con los paganos y los judíos son anticuadas, y, nosotros, los cristianos damos

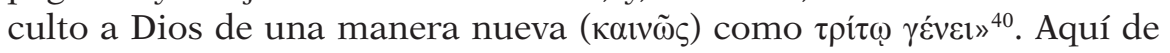

38 Sobre el texto, ver ibid., 242-244: «Christians are superior to the others in their understanding of divine power, in the character of their relationship with it, and in their search for the truth -not in their belonging to a disparate race-» (244). La apología también es atribuida a san Juan Damasceno, Sobre Barlaam y Joasaph, 27.

39 Así Gruen, 242-244, y Skarsaune, 262. Que no tiene sentido étnico queda más claro en la versión siriaca donde se dice que los bárbaros proceden de Crono y Rea y de sus otros dioses, y los griegos de Helena, que descendía de Zeus y de quien procede Eolo y Xutho, etc. Difícilmente se puede hablar de una «raza» de griegos, si los «progenitores» de esta raza son dioses. De hecho, el fragmento latino de Venecia introduce la cuestión con el término stirpes: "Compertum est nobis, o Rex, quatuor esse humani generis stirpes, quae sunt Barbarorum, Graecorum, Hebraeorum atque Christianorum».

40 Michel Cambe, ed., Kerygma Petri (CCSA 15; Turnhout: Brepols, 2003), 156-

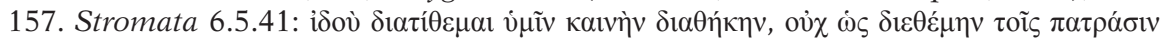

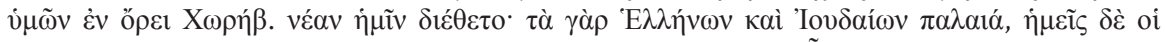

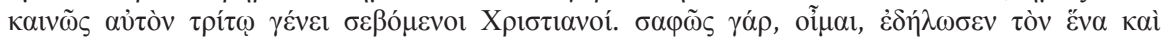

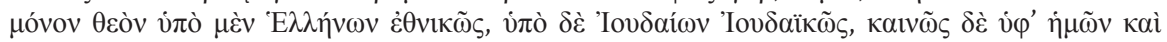

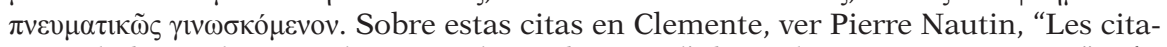
tions de la Prédication de Pierre dans Clement d'Alexandrie, Strom. VI 39-41", The Journal of Theolgial Studies New Series 25 (1974): 100-105; Antonio Orbe, Teologia 
nuevo tampoco es necesario entender $\gamma$ ćvo en sentido étnico ${ }^{41}$. Como explica Gruen, el texto gira sobre el culto a Dios y sobre cómo los cristianos no lo dan de la misma manera que los griegos o los judíos. Los cristianos lo hacen por mediación de Cristo. El pasaje se está refiriendo a una cuestión sobre el culto y las observancias, no va sobre razas; más

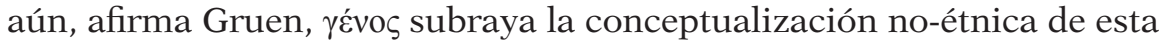
terminología. Por eso, concluye, podemos estar pensando desde nuestra perspectiva que un término es étnico cuando en realidad en los escritores cristianos se está refiriendo a prácticas, costumbres o modos de comportamiento que distinguen a los diferentes pueblos y es irrelevante en relación con características innatas o genéticas ${ }^{42}$.

Igualmente discutido es el sentido de la expresión latina tertium genus, tal como aparece en Tertuliano. Aquí el lenguaje étnico es ciertamente utilizado, pero de nuevo es cuestionable que implique una concepción étnica de los seguidores de Cristo. En su obra Ad nationes 1.8.5, el apologeta africano escribe que los cristianos no son una raza nueva desconocida; no tienen un tipo de dientes distinto ni órganos especiales para las pasiones incestuosas de las que se les acusa, como si fueran animales con ojos de perro (cynopennae) o criaturas de un solo pie (sciapodes) ${ }^{43}$.

de San Ireneo: Commentario al libro V del Adversus haereses (Madrid: BAC, 1988), 187-188. También Clemente hablará de griegos, judíos y cristianos (Strom . 5.14.58.4), en el sentido que de alguna forma está ya enunciado en 1Cor 10,32. Pero entiende

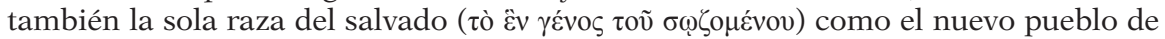
Dios. Ver además, Lona, 76-77, y Weidmann, 33-35.

41 De hecho, la traducción de Cambe es «type»: «les (pratiques) des Grecs et des Juifs sont périmées; c'est vous qui le vénérez d'une manière nouvelle, selon un troi-

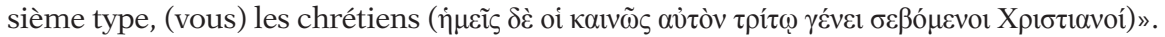
La de Montague Rhode James, The Apocryphal New Testament (Oxford: Clarendon Press 1924), 17, es «third generation (or race)». Ver Vollenweider, 298, n. 19.

42 Así Gruen, 242. Skarsaune, 262, es de la misma opinión y entiende, como en el caso de Arístides, que se trata de un escrito polémico y no étnico sobre el recto tipo ( $\gamma \varepsilon \dot{v}(\varsigma)$ ) de adoradores de lo divino. Gruen también señala que esto lo reconoció Harnack y remite a Cambe, 265-269 y a Buell, «Rethinking the Relevance of Race», 460461, quien en este caso prefiere entender la expresión como «tercer modo» o «tercera forma» en vez de "tercera raza» (aunque la autora sigue vinculándolo a la cuestión étnica y describiéndole como «ethnic reasoning»). Ver también Vollenweider, 298.

43 Cf. también Apologeticum 8.5: «Alia nos, opinor, natura; Cynopaene aut Sciapodes? Alii ordines dentium, alii ad incestam libidinem nervi?» Los cynopennae son criaturas míticas de cabeza de perro que habitaban en Persia o India (cf. Plinio, Hist. nat. 7.2.23). Los sciapodes son criaturas de un solo pie. Ver Isidoro, Etym. 11.3.23: «Sciopodum gens fertur in Aethiopia singulis cruribus et celeritate mirabili: quos 
En cambio, dirá en el Apologeticum, tienen que hacer negocios para vivir como todo el mundo, pues habitan entre los demás ciudadanos, viven sus mismas costumbres y tienen las mismas necesidades que los demás ${ }^{44}$. De estas afirmaciones parece deducirse que los seguidores de Cristo eran considerados tan extraños que habían llegado a ser definidos como un genus distinto, hasta el punto de ser llamados una tercer genus (en sentido despectivo), entendiendo por genus un tipo de especie humana diferente (Ad. Nat. 1.8.1: plane, tertium genus dicimur) ${ }^{45}$. El rechazo parecía total, pues, en otro lugar, el apologeta testimonia que en tiempos de persecución para los cristianos (quizá el 203 ó 204) en el circo se gritaba: "¿Por cuánto tiempo tendremos que sufrir esta tercera raza?» (Usque quo genus tertium?) (Scorp. 10). Se discute si este relato responde a la realidad o es fruto de la retórica de Tertuliano ${ }^{46}$, pero, cualquiera que fuera la realidad en el circo, lo que le interesa al de Cartago en el Ad Nationes es

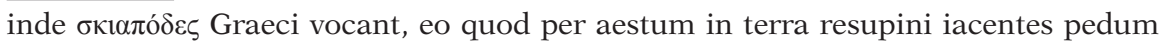
suorum magnitudine adumbrentur». Cf. al respecto Ingvild Saelid Gilhus, Animals, Gods and Humans: Changing Attitudes to Animals in Greek, Roman and Early Christian Thought (London: Routledge, 2006), 230-231.

44 Tertuliano, Apolog. 42: "Quo pacto homines vobiscum degentes, eiusdem victus, habitus, instructus, eiusdem ad vitam necessitatis? Neque enim Brachmanae aut Indorum gymnosophistae sumus, silvicolae et exules vitae. [2] Meminimus gratiam debere nos deo, domino, creatori; nullum fructum operum eius repudiamus: plane temperamus, ne[c] ultra modum aut perperam utamur. Itaque non sine foro, non sine macello, non sine balneis, tabernis, officinis, stabulis, nundinis vestris ceterisque commerciis cohabitamus in hoc saeculo. [3] Navigamus et nos vobiscum et militamus et rusticamur et mercamur; proinde miscemus artes, opera nostra publicamus usui vestro. Quomodo infructuosi videmur negotiis vestris, cum quibus et de quibus vivimus, non scio».

45 Adolf von Harnack, "Christians as a Third Race", en Mission and Expansion of Christianity in the First Three Centuries (trad. por James Moffatt del vol. 1 de la ed. de 1908; New York: Harper \& Brothers, 1962), 273-274, entiende que para Tertualiano la primera raza (genus primum) es la de griegos y romanos y todas las naciones cuyos dioses eran reconocidos, y la segunda (genus alterum) la de los judíos. Ver también Norbert Brox, "Non ulla gens non Christiana (zu Tertullian, Ad nat. 1, 8, 9 f.)”, Vigiliae Christianae 27 (1973): 46-49 y Andrew McGowan, "A Third Race or Not: The Rhetoric of Ethnic Self-Definition in Tertullian”, solo publicado en https://www.academia.edu/336990/A_Third_Race_or_Not_The_Rhetoric_of_Ethnic_ Self-Definition_in_Tertullian, consultado el 16.1.19.

${ }_{46}$ Así lo ve Vollenweider, 298, n. 22: «The data given by Tertullian, esp. his reference to the scene in the arena (Tert., Scorp. 10:10 [CCSL 2, 1089]), are much more a product of his sophisticated rhetoric than of historical facts - irrespective of Tertullian's own unhappiness with the designation of the tertium genus». 
mostrar lo absurdo de esa expresión. Y lo hace con fuerte ironía, argumentando que ese genus tertium también se podría dar entre los paganos en razón del sexo y no del culto ${ }^{47}$. Por otra parte, existe en Tertuliano una cierta tensión entre esta concepción negativa de genus y otra más neutra, pues refiere también en el Ad nationes 1,8.11 que los cristianos eran llamados tertium genus desde el punto de vista de la práctica religiosa y no del nacimiento («sed de superstitione tertium genus deputamur, non de natione, ut sint Romani, Iudaei, dehinc Christiani»). Se podría decir, por tanto, que para Tertuliano los cristianos son un genus, en lo que respecta al modo de dar culto a Dios -y en este sentido puede ser aplicado a ellos como natio y como superstitio-, pero no son un tertium genus, una raza diversa ${ }^{48}$. Como dice Gruen, la expresión «se refiere a la creencia, culto y práctica; en una palabra, a la religión, no a la etnicidad $»^{49}$. Cabe añadir que es posible que esta comprensión de los cristianos como un tipo diverso de personas haya surgido entre los no cristianos, a partir de la descripción que de ellos hace Suetonio: «Afflicti suppliciis Christiani, genus hominum, superstitionis novae et maleficae» (Nero 16.2). En todo caso las citas de Tertuliano no parecen hablar en términos raciales, sino que

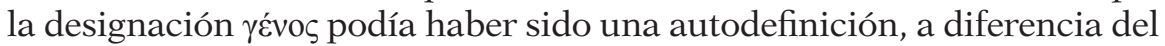
término cristiano, que probablemente surge entre los de fuera ${ }^{50}$.

47 En Ad Nat. 1.20.4 se refiere a que los paganos tenían entre ellos a eunucos o hermafroditas, que, al no ser ni hombre ni mujer, serían por tanto el tertium genus. En otro sentido, a veces en su obra se puede entender genus tertium como algo monstruoso, como cuando irónicamente dice en Tertul., de Virg. vel., 7.2: «Si el hombre es la cabeza de la mujer [Ef 5,22], por supuesto, también lo es de la virgen, de la que viene la mujer con la que se ha casado, a no ser que la virgen sea algo monstruoso con una cabeza propia, una tercera clase» (Si caput mulieris vir est, utique et virginis, de qua fit mulier illa quae nupsit, nisi si virgo tertium genus est monstruosum aliquod sui capitis). Cf. Harnack, 274.

48 Harnack, 277, piensa que solo después de mitad del siglo III se reconoce a esta raza de adoradores el título de natio, siendo el edicto de Decio la respuesta del Estado a esta concepción política que había surgido con Melitón y Orígenes. Concluye que el título de «nuevo pueblo» o "tercera raza» significa que había aparecido una nueva religión con las otras de las naciones ya existentes y con el judaísmo, y afirma que, utilizada por los cristianos, debió de haber servido como elemento diferenciador en relación a otras religiones (ibid., 278).

49 Gruen, 248.

50 Gruen, 247, afirma que pudo haber nacido en ámbito hostil contra los cristianos. En ibid., 238-239, ya había afirmado que el que Tácito y Suetonio califiquen respectivamente al cristianismo de superstitio y de genus indica que para los no cristianos no era una raza. Por eso sostiene que lo que nosotros podemos pensar que es 
Así las cosas, y a pesar de que $\gamma \varepsilon ́ v o \varsigma$ difícilmente tiene un sentido étnico en autores anteriores o contemporáneos a Diogn., los testimonios de la Apología de Arístides y del Kerigma Petri han llevado a que este término se haya comprendido en sentido étnico, en el marco de la «tercera raza» cuando en realidad es discutible ${ }^{51}$. Por tanto, lo ya visto invita a una considerar la posibilidad de una comprensión de otro ámbito. Para

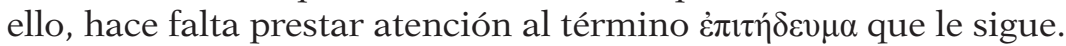

\section{2. ¿EsTILO DE VIDA?}

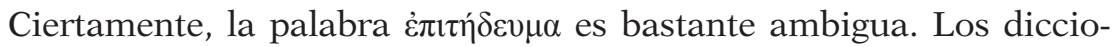

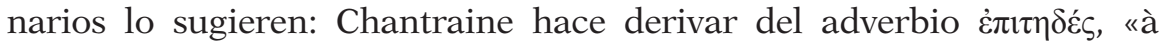

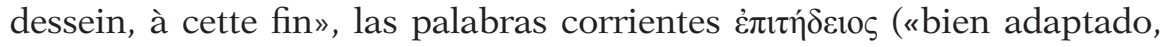
adecuado, útil»), para hablar de cosas y de personas, con empleos parti-

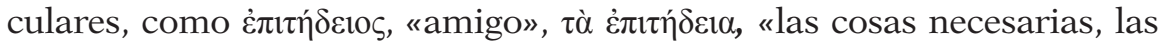

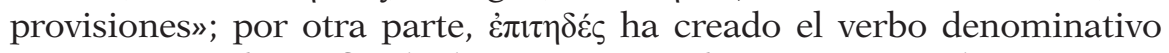
$\dot{\varepsilon} \pi \iota \tau \eta \delta \varepsilon v ́ \omega$ con el significado de "ocuparse, aplicarse a», cuya derivación en

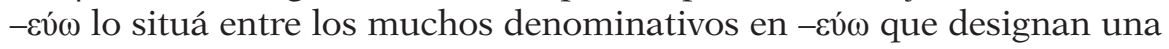

lenguaje de etnicidad, en cambio para ellos (1Pe y los romanos paganos) no lo era. En una línea parecida, Jefford, 70, entiende "tercera raza» como un reflejo del genus tertium de Tertuliano, como una designación que surge entre los oponentes al cristianismo basada en la manera que los cristianos daban culto. Ver también Lieu, «Identity Games», 60-61, quien se inclina a pensar que en Suetonio genus es más probable que se refiera a «clase», sin excluir connotaciones étnicas. Harnack, 274, n. 2, opina que el término nació espontánemente tanto entre los cristianos como entre sus oponentes. Horrell, “'Race', 'Nation', 'People”, 134, afirma: "Christian self-description in ethnic terms, drawing of course on Jewish identity discourse, and reinforced by the kinds of exclusivism that led to hostile criticism (Tacitus, Ann. 15.44), shaped outsiders' perceptions and descriptions». En este contexto Buell, "Race and Universalism", 445, declara: "We can begin to glimpse both the pervasiveness of ethnic reasoning and its strategic flexibility for early Christian self-definition».

51 Existe un curioso testimonio de Pseudo-Cipriano, De Pascha computus, 17, datado en el 243, donde el autor dice que «nosotros», los cristianos, somos identificados con «el tercer genus de la humanidad» («hic est gehennae ignis, in cuius similitudine caminus imperio regis septies tantum incensus per XL et VIIII cubita excreuit et aduersarios Ananiae Azariae et Misahel consumpsit, et ipsos tres pueros a Dei filio protectos [in mysterio nostro qui sumus tertium genus hominum] non uexauit»). Otro caso de «tercer

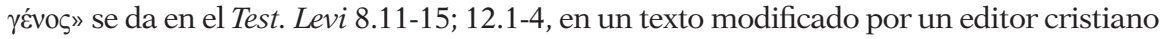
donde se afirma que la descendencia de Leví comprende tres $\gamma \varepsilon ́ v \eta$. Ver Skarsaune, 261, n. 45. 


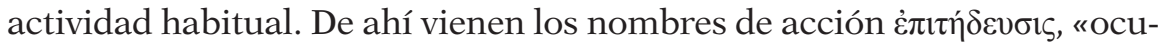

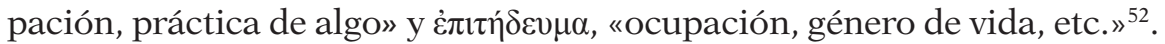

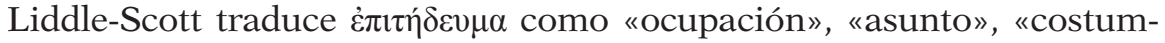
bre»; y añade que en plural también puede significar «hábitos»o "práctica de algo» (1. pursuit, business, custom, habits, practice of. 2. habit of

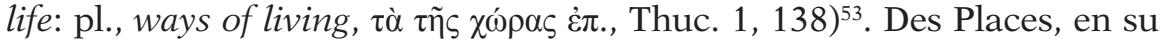

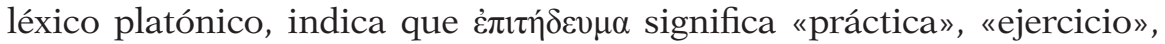
apareciendo habitualmente en plural con el significado de «costumbres»;

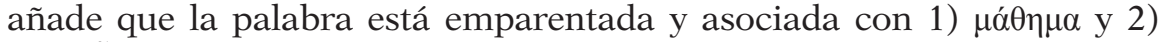

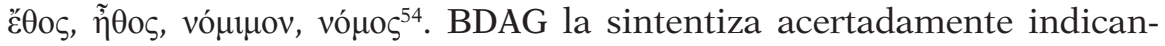
do que significa «that which one does with consistent purpose or habit, pursuit, way of living » ${ }^{55}$. Por otra parte, el término no ocurre en el Nuevo Testamento ni es recogido por Lampe's Patristic Lexicon ni por Sophocles' Greek Lexicon of the Roman and Byzantine Periods. Aun así, el vocabu-

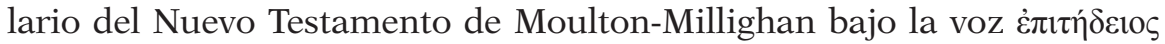
pone como ejemplo de غ̇ंı

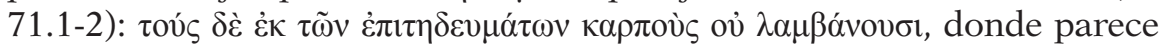
que los beneficios de unas estrellas quedan anulados por otros de manera que los jóvenes no recogen los frutos de sus «talentos» (en la traducción de Riley, aunque puede ser simplemente sus ocupaciones o negocios). En griego moderno ha pasado a significar «trabajo, profesión, ocupación».

En los LXX solo aparece en singular en Jdt 13,5, donde significa

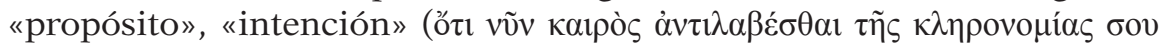

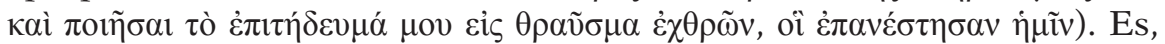
en cambio, frecuente en plural, significando «costumbres» $\mathrm{O}$ "modos de vida», aunque mayoritariamente tiene un sentido negativo (la Vulgata

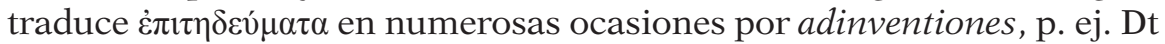
28,20; Jue 2,19; $1 \mathrm{Cr}$ 16,8; en cambio, es traducido por consuetudinem y

52 Pierre Chantraine, Dictionnaire étymologique de la langue grecque: histoire des mots, 4 vols. (Paris: Klincksieck, 1968-1977), s.v. غ̇ंı

53 Liddell, Greek-English Lexicon, s.v. غ̇ंı

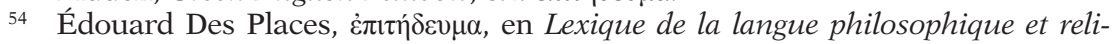
gieuse de Platon. Platon, Euvres complètes, vol. 14 (Paris: Les Belles Lettres, 1964). Por su parte, Lona, 78, n. 48, señala que en plural se encuentra en Platón como sinónimo de

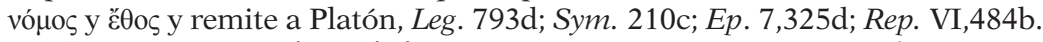

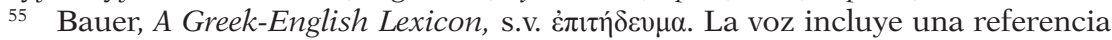
a Oenomaus en Eus., PE. 5.34.10, que se refiere al boxeo como una práctica divina

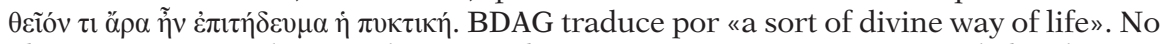
obstante, aquí puede entenderse simplemente como una «ocupación» de los dioses. 
morem en Lv 18,3). El término se da frecuentemente en Filón, también casi siempre en plural, en el sentido ya visto. En singular aparece en seis ocasiones como ocupación o práctica ${ }^{56}$. En Josefo, excepto en una ocasión (y en un caso dudoso), aparece siempre en plural con el significado de «esfuerzo, tarea, modo de actuar, conducta, comportamiento, modo de vida, modo de pensar» y «departamento, disciplina, habilidad, capa-

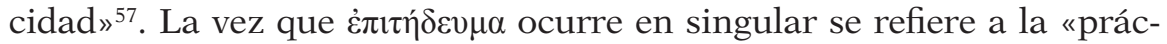
tica», en el sentido de habilidad de adornar el estilo con suavidad a la

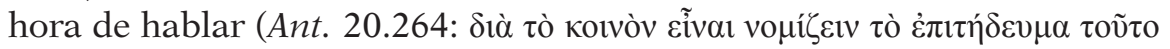

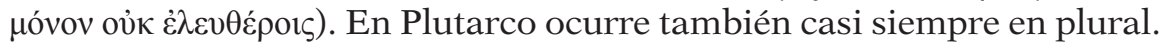
En singular aparece con el sentido habitual de «ocupación» o "práctica» ${ }^{58}$. A un nivel más filosófico se puede señalar el fragmento vinculado al de Queronea, Fragm. 198*, Scholia in Platonis Gorgiam, 462 e (307.12

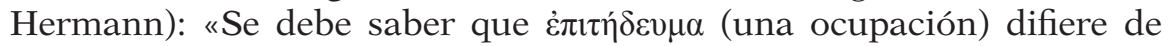

56 En singular: Spec. 1.343.5, en el sentido de una necesidad «habitual»; en Som. 2.21.3 y Flac. 3.4 como «negocio o ocupación»; en Legat. 231.1, como «práctica o costumbre»; en Ebr. 51.3 como «ocupación, estudio» referido a la filosofía y en Mut. 249.3,

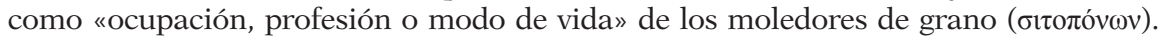
Ver también Peder Borgen et al., The Philo Index: A Complete Greek Word Index to the

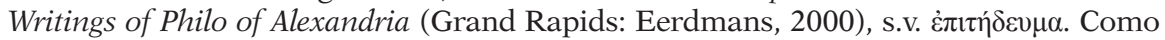
ejemplos del uso por parte de Filón de la palabra en plural en sentido de ley y costum-

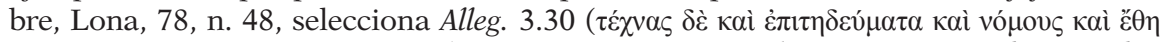

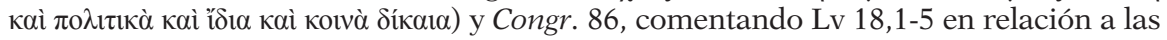

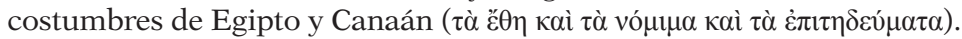

${ }^{57}$ Karl Heinrich Rengstorf, A Complete Concordance to Flavius Josephus, vol. 2

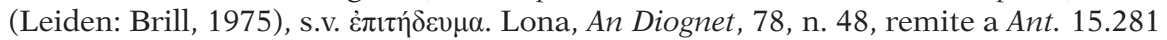
y $A p$. 2.187 para el uso plural, e indica también dos lugares que a su juicio se acer-

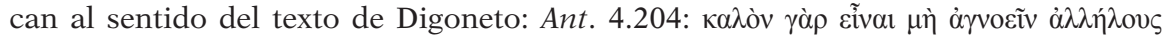

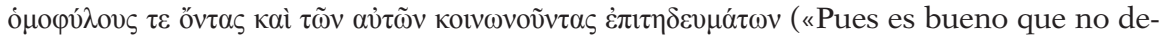
ban ignorarse entre sí, siendo miembros de la misma raza y compañeros en las mismas

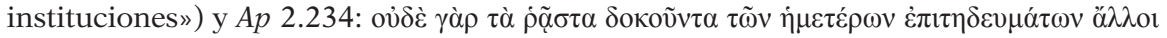

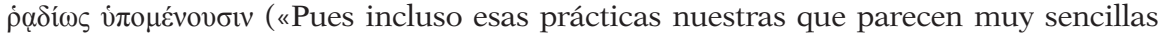
otros encuentran difíciles de tolerar»; luego Josefo enumera esas "prácticas»: servicio personal, dieta, disciplina para no caer en el capricho en relación a la comida, la bebida, a las cuestiones sexuales o a extravagancias, abstención de trabajo los sábados).

58 En Quaest. nat. 19 (Mor. 916C) significa "práctica»; en las Vidas (Them. 2.6) lo refiere a Mnesifilo que hacía de la filosofía su «ocupación» o «modo de vida»; en Ps.-Plut., Mus. (Mor. 1131E) como «la práctica de cultivar» la música; y en Quaest. conviv. 2.8 (Mor. 633A), «hábito» (en este caso, amor a la música o cazar). En De cur. 16 (Mor. 523b) habla de los que se dedicaban como «práctica» a acusar a otros de una actividad prohibida; y en Quaest. Plat. 3 (Mor. 1002b) se refiere a no estar esclavizado

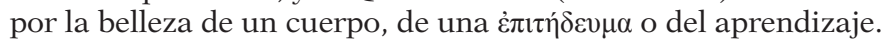




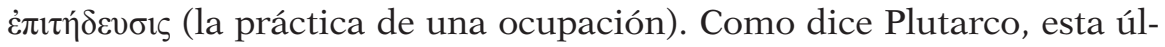
tima indica una actividad; la primera, en cambio, una quasi-sustancia»

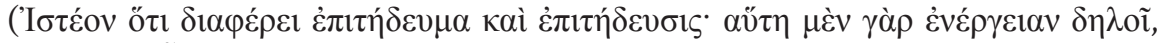

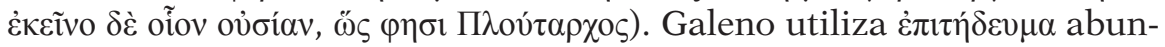
dantemente en plural, si bien, en proporción, es el autor del siglo II que más la utiliza en singular en el sentido de «ocupación» ${ }^{59}$.

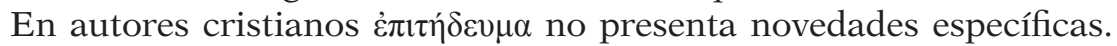
En Justino solo aparece en plural y se refiere a los «propósitos o ocupaciones» (Dial. 3.3.6) de la gente en general (las otras dos referencias son citas del A. T., Ez 20,19 y Sal 98,8, con el significado de acciones malvadas). Clemente de Alejandría también la utiliza a menudo en plural. Cuando ocurre en singular hace referencia a la «práctica» desmedida de la bebida (Pedag. 2.2.31.2; cf. 2.2.32.1 citando a Platón sobre lo mismo:

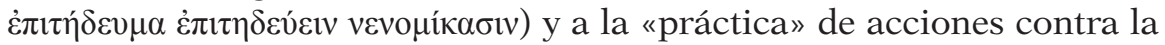
naturaleza (Pedag. 3.3.20.2) o la de las prostitutas (Pedag. 3.3.21.4). En Strom. 2.6.30.1, en cambio, habla de la fe como una "práctica», en sentido de hábito, que si fuese humana (ảv $\theta \rho \omega ́ \pi t v o v)$, «como algunos grie-

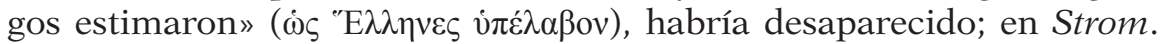

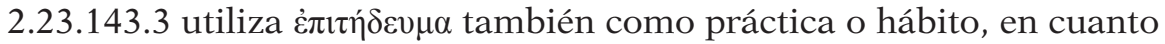
pasión, como una disposición baja y sucia en relación al matrimonio.

Más cercano a nuestro contexto estaría el apologista del siglo II Tacia-

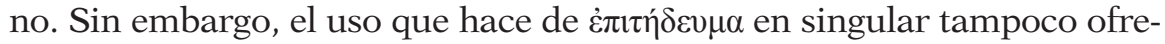
ce novedades. En el comienzo de su Oratio ad Graecos 1.1, acusando a los griegos de no ser capaces de crear algo nuevo sino solo de imitar, dice: «¿Qué práctica entre vosotros no tuvo su origen de los bárbaros?» (Пoĩov

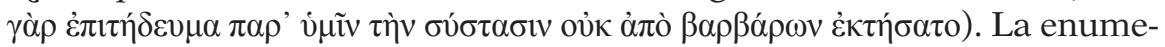

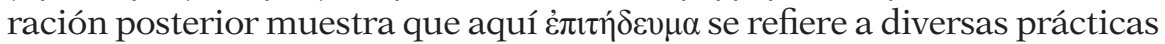
o profesiones (la adivinación por sueños, previsión del futuro por los astros y los vuelos de los pájaros, el arte de sacrificar, la astronomía, la magia, la geometría, el conocimiento de las letras). Más adelante, Orat. 28.1, al condenar la diversidad de legislaciones, se refiere a cómo entre los griegos se rehúye la unión con la propia madre mientras que los magos persas

59 Aparece en De sanitate tuenda 6.186.2 referida a la «utilidad» de un baño frío; en De naturalibus facultatibus 2.124, al hablar de la utilidad de la miel, se refiere a cómo actúa en cuerpos que están calientes por naturaleza, enfermedad, edad, estación del año u «ocupación»; en Thrasybulus 5.870.12 indica la "práctica» de los atletas. En singular, en Luciano, Abdic. 23.7 se refiere a la «ocupación» o profesión de los médicos y en Salt. 7.5 a la "práctica» u «ocupación» de la danza, que en 23.2 denomina

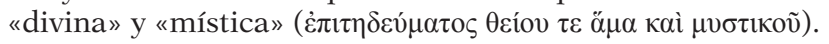




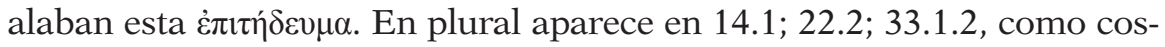
tumbres o prácticas, también de carácter filosófico $(31.1 ; 35.1)$.

Este sucinto repaso del término no permite determinar un sentido claro para nuestro pasaje de Diogn. Quizá lo más relevante es el predominio del uso en singular como ocupación o modo de ganarse la vida. En todo caso sugiere una actividad habitual cuya determinación debe venir dada por el contexto. Así las cosas, puede resultar útil rastrear el

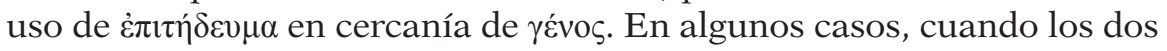
términos ocurren en un mismo contexto es porque responden a la nor-

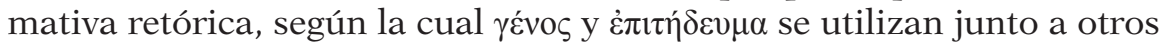
términos para definir a una persona. Por ej., Demóstenes, en Epit. 27.1, enumera las condiciones que hicieron posible a algunos morir noblemente por la patria: «nacimiento, educación, práctica de altos estándares de conducta, principios de la forma de gobierno» ( $\gamma \varepsilon ́ v o \varsigma, \pi \alpha 1 \delta \varepsilon i ́ \alpha, \chi \rho \eta \sigma \tau \tilde{\omega} v$

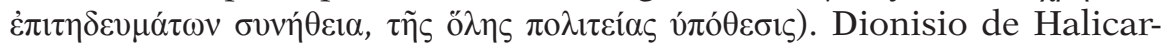
naso, en Lys. 9.1, entre los rasgos diferenciales de las personas sobre las que se debe hablar incluye «la edad, el trasfondo familiar, educación así como ocupación y modo de vida y otras cosas por las que se distinguen

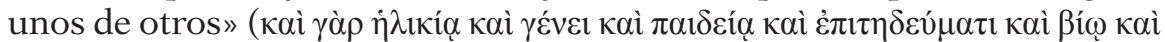

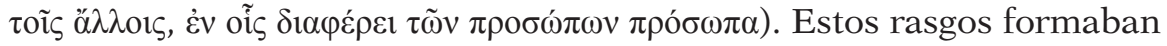
parte de la doctrina retórica sobre el discurso demostrativo, tal como lo recoge Aristóteles, en Rhet. 3.14 (1415b). En este tipo de discurso, dice el filósofo, el orador debe «lograr que el oyente crea que también él es elogiado, ya sea él mismo, o su linaje, o sus costumbres, o de cualquier

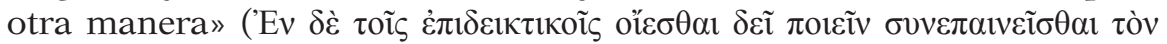

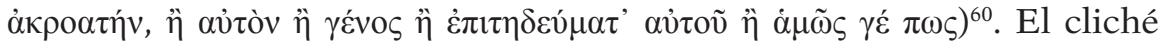
se encuentra de algún modo reflejado en el comienzo del Contra Euno-

60 El filósofo había hablado antes (1399b) de la identidad de las conclusiones según la identidad de las premisas, entendiendo غ̇ंı́́ $\delta \varepsilon v \mu \alpha$ como la actividad de filosofar:

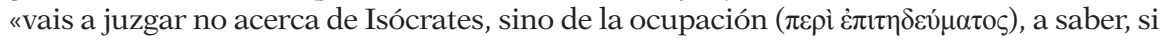

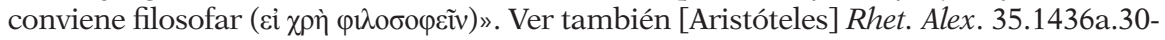
35 y Quintiliano, Inst. 3.8.48, quien afirma: «contribuye también mucho la personalidad de quien persuade, porque, si su vida pasada, el linaje, la edad y la fortuna hacen esperar cosas grandes... (ante acta vita si inlustris fuit aut clarius genus aut aetas aut fortuna adfert expectationem)», en donde genus podría también entenderse como «tipo de vida». En la misma línea Hermógenes, Peri eureseos 4.14 en relación al encomio dice:

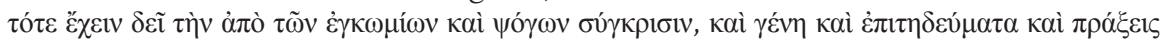

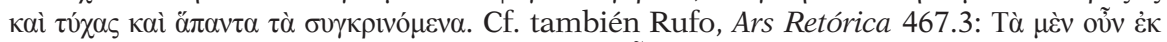

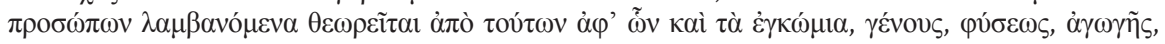

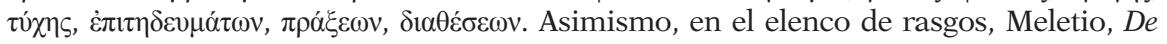


mium I de Gregorio de Nisa, donde se enuncian los temas que contiene el libro, entre los que se señala que «se tratará brevemente de los orí-

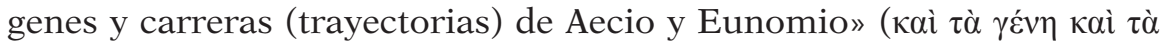

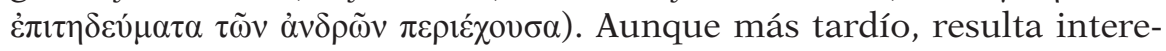
sante el sentido que aparece en Sinesio, Calu. 21, cuando escribe que

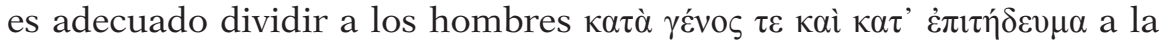
hora de argumentar correctamente qué corresponde a unos y a otros

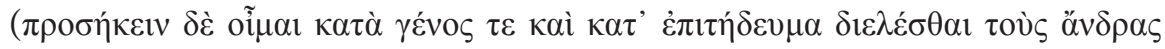

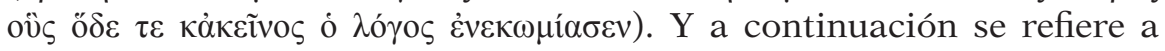

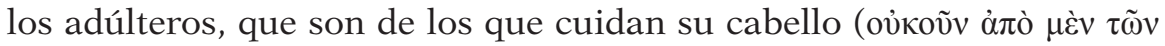

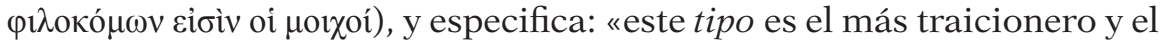

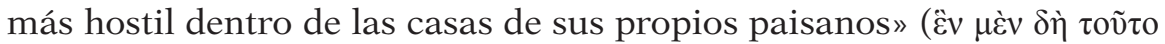

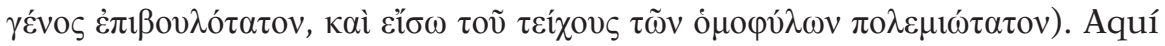
se observa claramente que $\gamma \varepsilon ́ v o \varsigma$ está desprovisto de connotación racial y sirve para indicar un tipo de personas, mientras que $\dot{\pi} \pi \imath \eta ́ \delta \varepsilon v \mu \alpha$ puede entenderse como costumbre.

Ciertamente, todos estos ejemplos no son concluyentes sobre el sentido que se le debe dar a la expresión de Diogn. 1. Por un lado queda claro el escaso uso singular de $\dot{\varepsilon} \pi \imath \dot{\delta} \delta \varepsilon \mu \alpha \alpha$, que tiene generalmente el sentido de práctica, ocupación o profesión, y por otro el uso retórico en combinación con $\gamma \varepsilon \dot{v}$ os, donde parece tener un cierto sentido étnico (si bien la misma naturaleza del discurso retórico sugiere que puede tener simplemente un carácter de fórmula). En todo caso, el sentido que aparece en Sinesio en combinación con غ̇ंı

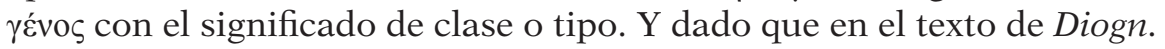

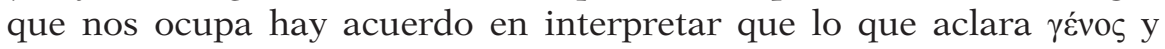

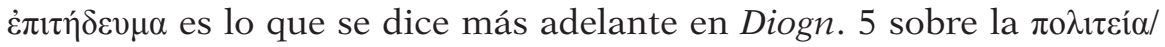

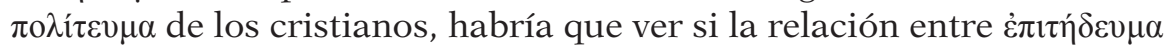
y estos términos ofrece alguna luz.

Por desgracia, tampoco son muchos los ejemplos en los que los dos

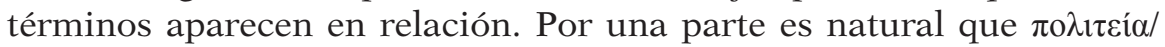
$\pi \circ \lambda i \tau \varepsilon v \mu \alpha$ se encuentren referidos a las costumbres (y por tanto con el

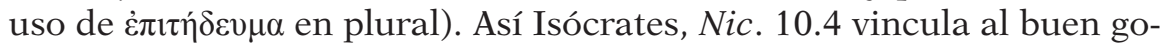

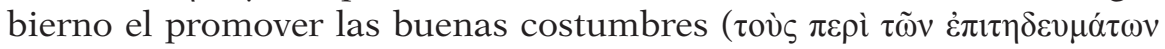

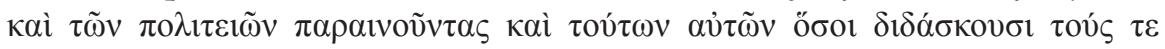

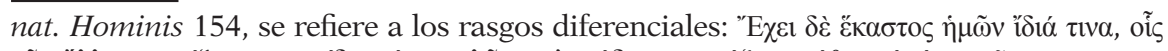

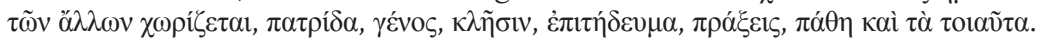




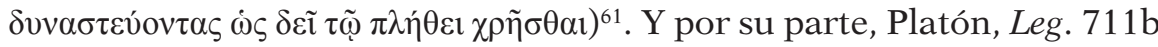
dice que el monarca necesita urgir a los ciudadanos a la práctica de

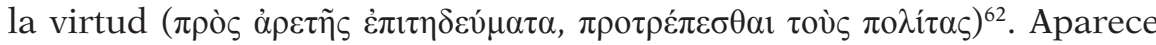
también en Dion. Halyc. Ant. Rom., 6.81.2, esta vez en singular cuando Valerio se queja de que se le ha acusado de una "práctica» impropia

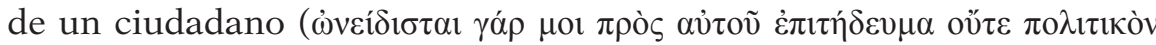

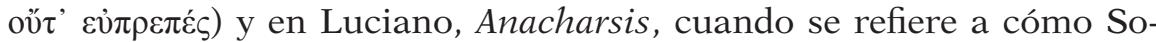

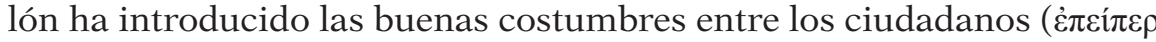

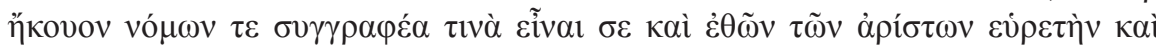

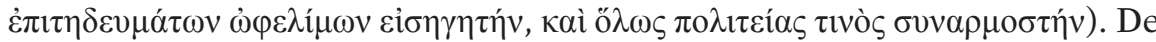
nuevo, hay que admitir que estos ejemplos tampoco aclaran demasiado. En cambio, hay un testimonio de Juan Crisóstomo, que a pesar de ser tardío respecto de Diogn., resulta iluminador. En su homilía sobre Luciano mártir el Crisóstomo relata el proceso que debió afrontar el santo e indica que en el interrogatorio al que fue sometido solo contestaba diciendo: "Soy cristiano». Y continúa predicando el obispo de Constantinopla: "Y cuando el verdugo dijo: «¿De qué país $(\pi \alpha \tau \rho i ́ \delta \alpha)$ eres?»,

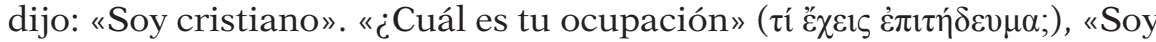

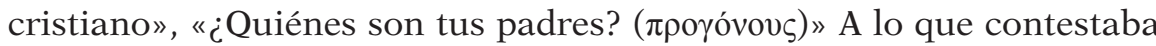
a todo: «Soy cristiano»». Tras unas consideraciones más el Crisóstomo concluye: "La persona que dice "Soy cristiano" ha revelado su país ( $\pi \alpha \tau \rho i ́ \delta \alpha)$, su estirpe ( $\gamma \varepsilon \dot{v} v \varsigma)$ y su ocupación $(\dot{\varepsilon} \pi \imath \tau \hat{\delta} \delta \varepsilon v \mu \alpha) »$. Y a continuación clarifica con textos de la Escritura cada uno de estos tres elementos:

«Déjame explicar cómo: el cristiano no tiene una ciudad en la tierra, sino la Jerusalén en el cielo. "Pues la Jerusalén celestial que es nuestra madre" dice la Escritura, "es libre" (Ga 4,26). El cristiano no tiene una ocupación terrena, sino que lleva un estilo de vida celestial

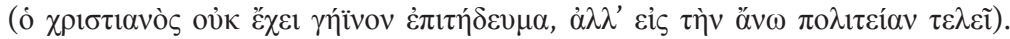

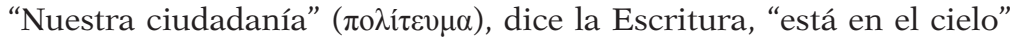
(Flp 3,20). El cristiano tiene como parientes y conciudadanos a to-

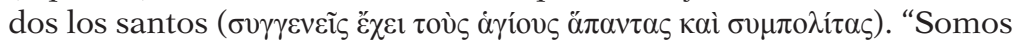

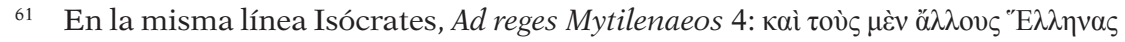

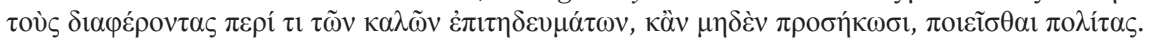

62 Y en Resp. [502d] «Aunque a duras penas, hemos terminado con esto, ahora nos queda por estudiar cómo y por medio de qué clase de estudio y experien-

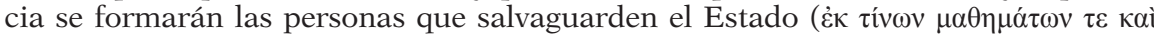

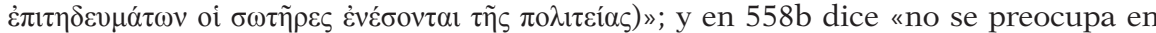
modo alguno del tipo de actividades al que se habrían dedicado los que van a la polí-

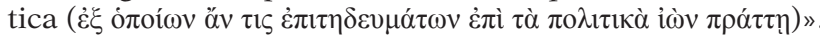




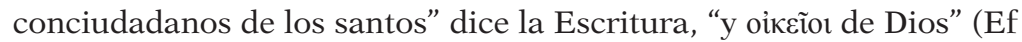
2,19). En consecuencia, con una palabra ha enseñado con precisión

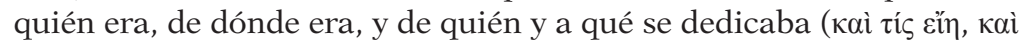

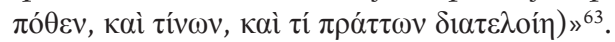

En parte, el trasfondo de los términos que emplea el Crisóstomo parece responder a lo que el discurso retórico exigía para describir a una persona y en este caso para tener información de ella: su origen, familia,

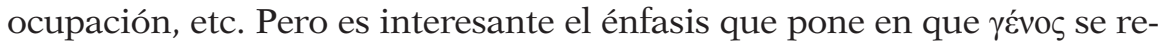

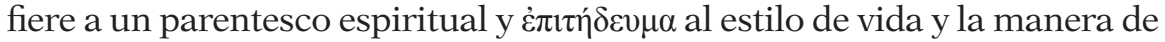
comportarse en la sociedad ${ }^{64}$. Esta comprensión sería aplicable a Diogn. 1, conforme a lo que más adelante se afirma en Diogn. 5-6 (con 7-8). En

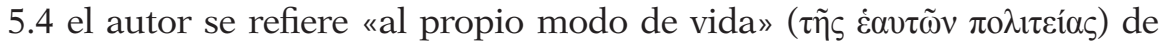
los cristianos en cuanto que no son distintos de otras personas, ni de su propio pueblo, ni de sus propias invenciones ${ }^{65}$. Los cristianos, dirá en 6.3,

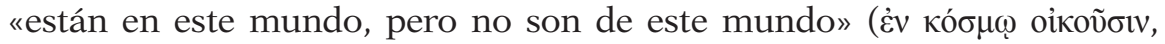

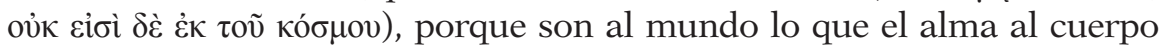
(Diogn. 6). Y esto es posible porque, para el autor de la carta, los discípulos de Cristo han recibido la revelación divina que está destinada a toda la humanidad y no solo a ellos (habla de los «hombres» en general: Diogn. $7.2,4 ; 8.1)$. No constituyen, por tanto, una «raza» particular.

63 Hom. in sanctum Lucianum martyrem, PG 50:525. Además, en sus homilías

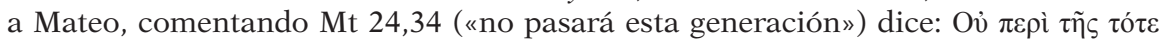

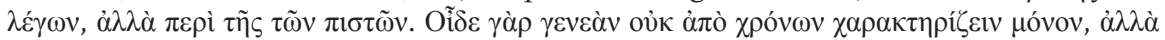

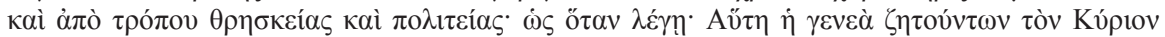
(«No hablaba [el Señor] de la generación de entonces, sino de la de los creyentes, porque [el Señor] sabe que una generación no se caracteriza sólo por el tiempo, sino también por la manera de su culto y de su vida, como cuando se dice: "esta es la generación de los que buscan al Señor” [Ps 24,6])» (Hom. in Matt. 77; PG 58:702).

64 En el pasaje de Taciano, Orat. 28.1, visto más arriba, donde condena la diversidad de legislaciones y habla de algunas غ̇ंı $\delta_{\varepsilon v ́} \mu \alpha \tau \alpha$, se refiere a cómo debería haber

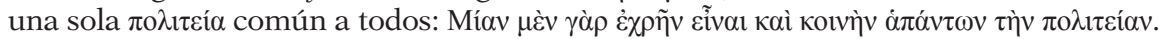

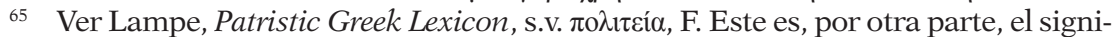

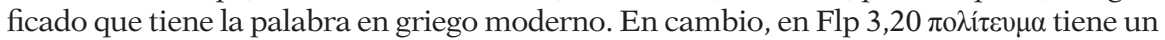
significado diferente. Se refiere a la «ciudadanía» en el cielo, como el hogar verdadero. Ver también Lieu, Neither Jew nor Greek?, 192-193, quien considera que $\pi$ ò $1 \tau \varepsilon i ́ \alpha$ puede considerarse fundacional para la identidad cristiana en Diogn. y que, según la autora, «implies a complete relativization of any local loyalty or identity». Ver también las observaciones de Doru Costache, "Christianity and the World in the Letter to Diognetus: Inferences for Contemporary Ecclesial Experience”, Phronema 27 (2012): 29-50. 


\section{CONCLUSIÓN}

El recorrido realizado muestra que, en Diogn. 1, una interpretación de

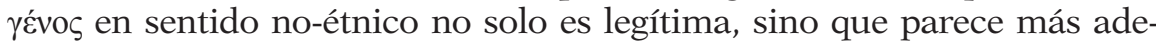
cuada: por una parte, porque el término lo permite, ya que uno de sus significados posibles es «tipo» o «clase»; y por otra, porque el que vaya explicitado por غ̇ंı

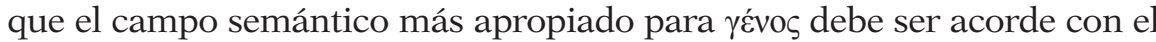

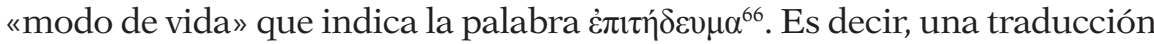
como «nueva raza o modo de vida» está poniendo en paralelo dos conceptos difícilmente comparables entre sí por medio de la conjunción. La traducción parece más bien presentar una alternativa entre términos no homogéneos. En cambio, si, tal como explica la carta después, ese modo

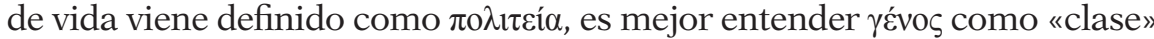
o «tipo de comportamiento» que como «raza» o "pueblo». Esta interpretación vendría confirmada por la contundencia con que Diogn. afirma que los cristianos no se distinguen de los demás: «No practican un tipo de vida

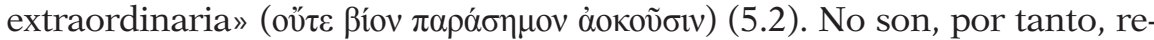
conocibles por los rasgos que distinguirían a un $\gamma \varepsilon ́ v o \varsigma$ entre otros ${ }^{67}$. En este sentido, la traducción de Lightfood-Harmer señalada al principio («new development or interest»), sin ser precisa del todo, estaría más cercana a esta comprensión. Así las cosas, se podría pensar que ĭ más que un aut o vel habría que entenderlo como un sive. Estaría introduciendo una alternativa que resulta más apropiada para expresar lo que se acaba de decir.

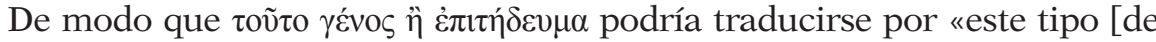
comportamiento] o más bien (o más aún, o para ser preciso) modo de vivir» ${ }^{68}$. Esta interpretación resuelve muchas de las dificultades y conflictos que encuentran quienes combinan una comprensión de yévos en términos

66 Jefford, 201, lo ve en continuidad con el más antiguo modo de identificar al cristianismo como «camino del Señor» (Hch 18,25) y "camino de Dios» (Hch 18,26).

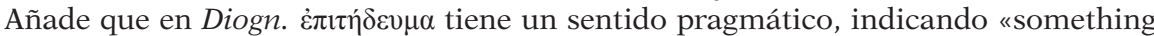
more like divine "life-style"».

67 Como afirma Enrico Norelli, A Diogneto. Introduzione, traduzione e note (Milano: Paoline, 1991), 90, «benché i cristiani siano un genos, non si distinguono per le caratteristiche proprie dei genê». Sin embargo, entiende que "este modo de vida particular» probablemente hace referencia a los elementos etnográficamente distintivos de un genos. En su opinión, esta comprensión de genos le viene a Diogn. de las fuentes que utiliza (Kerygma Petri y Arístides).

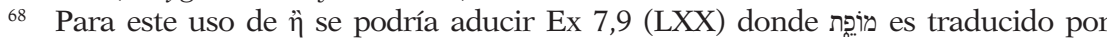

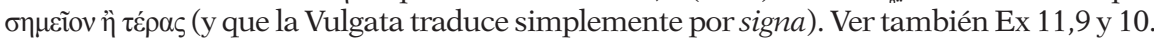


étnico-raciales y lo que afirma la misma carta. Es el caso, por ejemplo, de la obra de Buell, para quien lo que se afirma en Diogn. sería un modelo de situación borrosa entre categorías étnico-raciales y cívicas, pero que no se sabe muy bien cómo compaginar. Comentando Diogn. 5.4-5.9, afirma:

«This text initially links Christians with a race (genos), but when we ask what this means, we find the notion of citizenship at the center. For the Epistle to Diognetus, this defining feature makes it possible to portray Christianness in at least partially universalizing terms-as an identity that, like other forms of citizenship, is potentially accessible to all free male people, something that sets Christians apart yet allows them to otherwise "fit" into the status quo» ${ }^{69}$.

La autora parece interpretar que los cristianos son un $\gamma \varepsilon ́$ vo

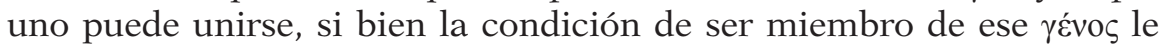
hace ser forastero-residente y extraño en medio de otros pueblos. Es decir, el autor de la carta estaría diciendo que los cristianos son un pueblo pero no tienen los rasgos distintivos característicos de un grupo étnico o pueblo (vestido, dieta y otros lugares comunes), pues de ellos se afirma que no se distinguen de los demás «ni por su tierra ni por su habla ni por sus costumbres» (5.1), «ni habitan ciudades exclusivas suyas, ni hablan una lengua extraña, ni llevan un género de vida aparte de los demás» (5.2), sino que habitan «ciudades griegas o bárbaras, según la suerte que a cada uno le cupo, y adaptándose en vestido, comida y demás género de vida a los usos y costumbres de cada país» (5.4). Pero si esto es así, ¿cómo es posible una comprensión étnica sin los rasgos característicos que definen a un grupo étnico ${ }^{70}$. Porque lo que sostiene Diogn. es que los cristianos se distinguen de los demás no por unos rasgos étnicos sino por su conducta moral: «Dan muestras de un tenor de peculiar conducta, admirable, y, por confesión de todos, sorprendente» (5.4b). Y es este modo de comportarse el que les concede un estatus de extranjeros: «Habitan sus propias patrias, pero como forasteros: toman parte en todo

\footnotetext{
69 Buell, Why This New Race, 31. Cf. Dunning, 68.

70 Como plantea Lieu, Neither Jew nor Greek?, 200: «The practical problem, therefore, is what is a third race without an identity? Again, Christian self-designation as a race, which in the second century we also find in the Martyrdom of Polycarp and in Melito of Sardis, as well as in the Kerygma Petri excerpted by Clement of Alexandria, is paralleled by and perhaps dependent on the Jewish self-ascription common in the Maccabean literature. Yet this only underscores the contrast and the dilemma, for what constitutes a "race"? Is an identity which subsumes and so obviates the identity of Jew or Greek a possibility?».
} 
como ciudadanos y todo lo soportan como extranjeros; toda tierra extraña es para ellos patria, y toda patria, tierra extraña» (5.5).

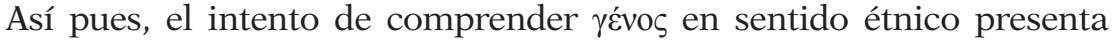
tensiones difíciles de resolver. En todo caso, admitiendo que $\gamma \varepsilon \dot{v} v \varsigma$ haga referencia a un grupo de personas concreto, con una connotación de descendencia común, sería mejor interpretarlo como referencia a un estado de parentesco espiritual (en el sentido que luego lo entiende Juan Crisóstomo en el texto arriba señalado). En castellano los términos que podrían reflejar esta comprensión serían «linaje», en la acepción de «clase o condición de una cosa»; o, incluso mejor, «estirpe», como cuando en términos jurídicos se utiliza para designar un conjunto formado por la descendencia de un sujeto a quien esta descendencia representa y ocupa su lugar. No obstante, la presencia de $\dot{\varepsilon} \pi \imath \eta ́ \delta \varepsilon v \mu \alpha$ sugiere que la novedad que tiene en mente Diogneto según el autor de la carta no es la de un grupo con una identidad propia, como podía ser la del pueblo judío o incluso la de los adeptos a una religión. Es más bien la de un modo de ser en la sociedad. No se trata de una cuestión racial sino de una manera de estar en el mundo.

\section{REFERENCIAS}

Bauer, W., William F. Arndt y F. William Gingrich. A Greek-English Lexicon of the New Testament and Other Early Christian Literature. $3^{\text {rd }}$ ed. Chicago: University of Chicago Press, 2000.

Borgen Peder, K. Fuglseth, \& R. Skarsten. The Philo Index: A Complete Greek Word Index to the Writings of Philo of Alexandria. Grand Rapids: Eerdmans, 2000.

Brett, Mark G., ed. Ethnicity and the Bible. Boston: Brill, 1996.

Brox, Norbert. «Non ulla gens non Christiana (zu Tertullian, Ad nat. 1, 8, 9 f.)». Vigiliae Christianae 27 (1973): 46-49. https://doi.org/10.2307/1583287

Buell, Denise K. «Rethinking the Relevance of Race for Early Christian Self-Definition». Harvard Theological Review 94 (2001): 449-476.

-. «Race and Universalism in Early Christianity». Journal of Early Cristian Studies 10 (2002): 429-468.

-. Why This New Race: Ethnic Reasoning in Early Christianity. New York: Columbia University Press, 2005.

Byron, Gay L. Symbolic Blackness and Ethnic Difference in Early Christian Literature. NewYork: Routledge, 2002.

Cambe, Michel. Kerygma Petri. CCSA 15. Turnhout: Brepols, 2003. 
Chapa, Juan. "Sobre la relación laos-laikos". En La misión del laico en la Iglesia y en el mundo, dirigido por José Luis Illanes et al., 197-212. Pamplona: Eunsa, 1987.

Chantraine, Pierre. Dictionnaire étymologique de la langue grecque: histoire des mots, 4 vols. Paris: Klincksieck, 1968-1977.

Cohen, Shaye J. D. Why Aren't Jewish Women Circumcised? Gender and Covenant in Judaism. Berkeley: University of California Press, 2005.

Costache, Doru. "Christianity and the World in the Letter to Diognetus: Inferences for Contemporary Ecclesial Experience". Phronema 27 (2012): 29-50.

Des Places, Édouard. Lexique de la langue philosophique et religieuse de Platon. Platon, Euvres complètes. Vol. 14. Paris: Les Belles Lettres, 1964.

Duling, Dennis C. "2 Corinthians 11:22: Historical Context, Rhetoric, and Ethnic Identity". En The New Testament and Early Christian Literature in Greco-Roman Context: Studies in Honor of David E. Aune, editado por John Fotopoulos, 63-87. Novum Testamentum, Supplements 122. Leiden: Brill, 2006. https://doi.org/10.1163/ej.9789004143043.i-465.26

Dunning, Benjamin H. Aliens and Sojourners: Self as Other in Early Christianity, Divinations: Rereading Late Ancient Religion. Philadelphia: University of Pennsylvania Press, 2009.

Gilhus, Ingvild Saelid. Animals, Gods and Humans: Changing Attitudes to Animals in Greek, Roman and Early Christian Thought. London: Routledge, 2006. https://doi.org/10.4324/9780203964798

Gruen, Erich S. "Recensión al libro de D. K. Buell, Why This New Race". Catholic Biblical Quaterly 72 (2010): 365-367.

-. "Christians as a 'Third Race'". En Christianity in the Second Century. Themes and Developments, editado por James Carleton Paget \& Judith Lieu, 235-249. Cambridge: Cambridge University Press, 2017. https://doi. org/10.1017/9781316691601.015

Hall, Edith. Inventing the Barbarian: Greek Self-Definition through Tragedy. Oxford: Oxford University Press, 1991.

Hall, Jonathan M. Ethnic Identity in Greek Antiquity. Cambridge: Cambridge University Press, 1997.

Harnack, Adolf von. "Christians as a Third Race". En Mission and Expansion of Christianity in the First Three Centuries. Traducido por James Moffatt del vol. 1 de la ed. de 1908. 266-278. New York: Harper \& Brothers, 1962.

Hartog, Paul. Polycarp's Epistle to the Philippians and the Martyrdom of Polycarp. Oxford: Oxford University Press, 2013.

Horrell, David G. “'Race', 'Nation', 'People': Ethnic Identity-Construction in 1 Peter 2.9”. New Testament Studies 58 (2011): 123-143 (= Becoming a 
Christian. 133-163. London: Bloomsbury T\&T Clark, 2013). https://doi. org/10.1017/S0028688511000245

-. Becoming a Christian. Essays in 1 Peter and the Making of Christian Identity. London: Bloomsbury T\&T Clark, 2013.

Horrell, David G., \& Katharine M. Hockey, eds. Ethnicity, Race, Religion. Identities and Ideologies in Early Jewish and Christian Texts, and in Modern Biblical Interpretation. London: T\&T Clark, 2018. http://dx.doi. org/10.5040/9780567677334

Hutchinson, John, \& Anthony D. Smith, eds. Ethnicity. Oxford: Oxford University Press, 1996.

Isaac, Benjamin. The Invention of Racism in Classical Antiquity. Princeton: Princeton University Press, 2004.

James, Montague Rhode. The Apocryphal New Testament. Oxford: Clarendon Press 1924.

Jefford, Clayton N. The Epistle to Diognetus (with the Fragment of Quadratus). Introduction, Text and Commentary. Oxford: Oxford University Press, 2013.

Johnson, Aaron P. "Recensión al libro de Buell Why This New Race". Bryn Mawr Classical Review 2006.02.31 http://bmcr.brynmawr.edu/2006/ 2006-02-31.html (consultado el 28.9.18).

Johnson, Aaron P. Ethnicity and Argument in Eusebius' Praeparatio Evangelica. Oxford Early Christian Studies. Oxford: Oxford University Press, 2006.

Jones, C. P. "ع̌ $\theta v o \varsigma$ and $\gamma \varepsilon \dot{v} o \varsigma$ in Herodotus". The Classical Quaterly 46 (1996): 315-320.

Jones, Siân. The Archeology of Ethnicity: Constructing Identities in the Past and the Present. New York: Routledge, 1997.

Lampe, Peter. From Paul to Valentinus: Christians at Rome in the First Two Centuries. Traducido por Michael Steinhauser. Minneapolis: Fortress Press, 2003.

Liddell, H. G., Robert Scott \& H. Stuart Jones. Greek-English Lexicon. $9^{\text {th }}$ ed. with revised supplement. Oxford: Clarendon Press, 1996.

Lieu, Judith M. "Race of the God-Fearers". The Journal of Theological Studies New Series 46 (1995): 483-501 (= Neither Jew nor Greek? 66-85. London: Bloomsbury, $\left.{ }^{2} 2016\right)$. https://doi.org/10.1093/jts/46.2.483

-. Image and Reality: The Jews in the World of the Christians in the Second Century. London: T\&T Clark, 1996.

-. "The Forging of Christian Identity". Mediterranean Archaeology 11 (1998): 71-82 (= "The Forging of Christian Identity and the Letter to Diognetus". En Neither Jew nor Greek? 185-203. London: Bloomsbury, $\left.{ }^{2} 2016\right)$.

-. Christian Identity in the Jewish and Graeco-Roman World. New York: Oxford University Press, 2004. 
-. Neither Jew nor Greek? Constructing Early Christianity. Edinburgh: T\&T Clark/ Continuum, 2002, 2. ${ }^{\mathrm{a}}$ ed. London: Bloomsbury, 2016.

-. "Identity Games in Early Christian Texts: The Letter to Diognetus". En Ethnicity, Race, Religion. Identities and Ideologies in Early Jewish and Christian Texts, and in Modern Biblical Interpretation, editado por David G. Horrell \& Katharine M. Hockey, 59-72. London: T\&T Clark, 2018. http://dx.doi.org/10.5040/9780567677334.0010

Lona, Horacio E. An Diognet. Übersetzt und erklärt. Freiburg: Herder, 2001.

Lust, J., Erik Eynikel \& Katrin Hauspie. Greek-English Lexicon of the Septuagint. Stuttgart: Deutsche Bibelgesellschaft, 1992-1996.

McGowan, Andrew. "A Third Race or Not: The Rhetoric of Ethnic SelfDefinition in Tertullian". https://www.academia.edu/336990/A_Third_ Race_or_Not_The_Rhetoric_of_Ethnic_Self-Definition_in_Tertullian (consultado el 28.9.18).

Mitchell, Margaret M. "Recensión al libro de Buell Why This New Race". History of Religions 48 (2008): 173-177.

Moss, Candida. "On the Dating of Polycarp: Rethinking the Place of the Martyrdom of Polycarp in the History of Christianity". Early Christianity 1 (2010): 539-574. https://doi.org/10.1628/186870310793597051

Nautin, Pierre. "Les citations de la Prédication de Pierre dans Clement d'Alexandrie, Strom. VI 39-41". The Journal of Theolgial Studies New Series 25 (1974): 100-105. https://doi.org/10.1093/jts/xxv.1.98

Norelli, Enrico. A Diogneto. Introduzione, traduzione e note. Milano: Paoline, 1991.

Orbe, Antonio. Teologia de San Ireneo: Commentario al libro V del Adversus haereses. Madrid: BAC, 1988.

Rengstorf, Karl Heinrich. A Complete Concordance to Flavius Josephus. Vol. 2. Leiden: Brill, 1975.

Skarsaune, Oskar. "Ethnic Discouse in Early Christianity". En Christianity in the Second Century. Themes and Developments, editado por James Carleton Paget \& Judith Lieu, 250-264. Cambridge: Cambridge University Press, 2017. https://doi.org/10.1017/9781316691601.016

Vollenweider, Samuel. "Are Christians a New 'People'?: Detecting Ethnicity and Cultural Friction in Paul's Letters and Early Christianity". Early Christianity 8 (2017): 293-308. https://doi.org/10.1628/18687031 7X15017545210198

Weidmann, Frederick W. “To Sojourn' or 'To Dwell'?: Scripture and Identity in the Martyrdom of Polycarp". En Reading in Christian Communities: Essays on Interpretation in the Early Church, editado por Charles A. Bobertz \& David Brakke, 29-40. Notre Dame, IN: University of Notre Dame Press, 2002. 